\title{
Recombinant BCG overexpressing a STING agonist elicits trained immunity and improved antitumor efficacy in non-muscle invasive bladder cancer
}

Alok Kumar Singh ${ }^{1}$, Monali Praharaj ${ }^{1,4}$, Kara A Lombardo ${ }^{2}$, Takahiro Yoshida ${ }^{3}$, Andres Matoso $^{4}$, Alex S. Baras ${ }^{4}$, Liang Zhao ${ }^{5}$, Pankaj Prasad ${ }^{1}$, Jonathan D. Powell ${ }^{5}$, Max Kates ${ }^{2}$, David McConkey ${ }^{2}$, Drew M. Pardoll ${ }^{5}$, William R. Bishai ${ }^{1, *}$ Trinity J. Bivalacqua ${ }^{2, *}$

${ }^{1}$ Johns Hopkins University, School of Medicine, Department of Medicine, Center for Tuberculosis Research, Baltimore, USA

${ }^{2}$ Johns Hopkins University, School of Medicine, Department of Urology, Baltimore, USA

${ }^{3}$ Department of Urology, Hyogo Prefectural Nishinomiya Hospital, Japan, 6620918

${ }^{4}$ Department of Pathology, The Johns Hopkins University, Baltimore, USA

${ }^{5}$ The Bloomberg-Kimmel Institute for Cancer Immunotherapy at Johns Hopkins, Baltimore, USA.

\section{Correspondence:}

*Joint corresponding authors

Trinity J. Bivalacqua, MD, PhD

Johns Hopkins Medical Institutions

The James Buchanan Brady Urological Institute

600 N. Wolfe Street, Marburg 409

Baltimore, MD 21287

Tel: 443-287-0385

Email: tbivala1@jhmi.edu

\author{
William Bishai, MD, PhD \\ JHU School of Medicine \\ CRB2, Room 108 \\ 1550 Orleans Street \\ Baltimore, MD 21287 \\ e-mail: wbishai@jhmi.edu
}




\begin{abstract}
:
BCG remains first-line therapy for non-muscle invasive bladder cancer (NIMBC) but its mechanism of action is not fully understood nor is its efficacy complete. We engineered a recombinant BCG (BCG-STING) that releases increased levels the STING agonist, c-di-AMP. Compared with BCG, BCG-STING demonstrated superior antitumor efficacy in models of NMIBC, more potent pro-inflammatory cytokine responses, greater myeloid cell reprogramming (M1 shift) associated with enhanced epigenetic and metabolomic changes and enhanced Teffector infiltration, all favoring antitumor immunity. These findings support the local induction by BCGSTING of enhanced and remodeled innate immune responses, sometimes termed trained immunity, and ultimately enhanced T cell immunity. They reveal that STING pathway activation is a proximal trigger in trained immunity remodeling, which may be a central mechanism for both BCG and BCG-STING antitumor activity in NMIBC.
\end{abstract}

\title{
SIGNIFICANCE:
}

This study demonstrates that the antitumor efficacy of BCG during bladder cancer is mediated via myeloid cell "training" in which BCG leads to epigenetic modifications accompanied by metabolic and cellular reprogramming changes which promote antitumor immunity. Using a recombinant BCG strain which delivers high levels of a STING agonist, we further demonstrate that STING pathway stimulation strongly enhances myeloid cell training as well as antitumor potency. 


\section{INTRODUCTION:}

Bacillus Calmette-Guérin (BCG)--the only FDA-approved bacterial agent for cancer immunotherapy--has been in use for the treatment of high-risk non-muscle invasive bladder cancer (NMIBC) as a first-line therapy since the late 1970s. More than one-third of patients with NMIBC will experience tumor recurrence after receiving BCG, and these patients have limited options other than removal of the bladder and creation of a urinary diversion - a major life-altering event. Thus, there is a significant unmet need for improved versions of BCG that provide superior response rates and prevent disease progression ${ }^{1}$. At the current time intravesical options for bladder preserving therapy remain limited.

Following bladder instillation, BCG induces a local inflammatory response accompanied by infiltration of granulocytes, macrophages, natural killer (NK) cells, dendritic cells (DCs), and $\mathrm{CD}^{+}$and $\mathrm{CD}^{+} \mathrm{T}$ cells, accompanied by release of pro-inflammatory cytokines including IL-6, TNF- $\alpha$, and IFN- $\gamma^{2-7}$. However, the specific immune mechanisms leading to BCG-mediated tumor eradication as well as BCG-resistance are not well-understood. BCG has been found to impart potent heterologous protection against non-related viral and bacterial infections; it elicits this protection via an innate immune memory mechanism known as trained immunity ${ }^{8-11}$. Trained immunity is characterized by metabolic, epigenetic and transcriptional reprogramming of both myeloid and lymphoid lineages ${ }^{12-15}$ but it has not been extensively studied as an antitumor mechanism during BCG immunotherapy for NMIBC.

Recent studies have implicated the cytosolically located, double strand DNA-sensing receptor cyclic GMP-AMP synthase (cGAS) and its downstream signaling effector, stimulator of interferon genes (STING), in a key innate immune response pathway known as the cytosolic surveillance pathway (CSP), which responds to DNA and cyclic dinucleotides (CDN) aberrantly present in the cytosol ${ }^{16-19}$. CDNs can come from intracellular bacteria that synthesize and secrete them, or alternatively when DNA-activated cGAS catalyzes the formation of 2'3'-cGAMP, a CDN that is a potent STING agonist. Activation of STING leads TBK1 activation, which in turn 
stimulates canonical IRF3-driven Type I interferon and IKK-driven NF-kB-mediated innate immune responses, including elevated dendritic cell priming and recruitment of antigen-specific T-effector (Teff) cells $\mathrm{s}^{20-23}$. Small molecule STING agonists elicit potent pro-inflammatory responses, and correspondingly have shown significant efficacy as cancer immunotherapies ${ }^{24,25}$.

BCG harbors an endogenous diadenylate synthase gene called $\operatorname{dis} A$, and the microbe naturally releases small amounts of the STING agonist c-di-AMP ${ }^{18,26}$. We hypothesized that this low-level engagement of STING may contribute to BCG's antitumor efficacy in NMIBC. Consequently, sought to determine whether enhanced activation of STING may augment BCGmediated trained immunity in preclinical models of NMIBC. To address these questions, we tested a recombinant disA-overexpressing BCG strain called BCG-disA-OE for its antitumor potency and ability to elicit trained immunity in urothelial cancer models.

\section{RESULTS}

\section{BCG-disA-OE shows improved efficacy against urothelial cancer in two animal models.}

BCG-disA-OE is a genetically-engineered BCG strain in which disA is fused to a strong promoter, leading to a 300 -fold overexpression of dis $A$ and a 15 -fold increase in production of c-di-AMP (Fig. S1a) ${ }^{18}$. Compared with wild type BCG (BCG-WT), BCG-disA-OE elicits significantly increased STING activation in macrophages as measured by IRF3 induction (Fig. S1b). To evaluate its NMIBC antitumor efficacy we tested BCG-disA-OE delivered intravesically in the rat $N$-methyl- $N$-nitrosourea (MNU) model of $\mathrm{NMIBC}^{27-29}$. In this model urothelial dysplasia develops at week 14 after the first intravesical instillation of MNU and by week 24 rats display a heterogeneous NIMBC including carcinoma-in-situ (CIS), papillary Ta, or higher-grade T1-T2 urothelial carcinoma with histopathologic and immunophenotypic features similar to those observed in human NMIBC (Fig. 1a). Rats treated with weekly intravesical BCG-disA-OE or BCG- 
WT from week 18-23 were sacrificed for bladder cytokine expression changes and histopathologic staging. As shown in Fig.1b, compared with BCG-WT, BCG-disA-OE elicited significantly increased levels of IFN- $\beta$, IFN- $\gamma$, TNF- $\alpha$, IL-1 $\beta$, CXCL 10, MCP-1, MIP- $1 \alpha$, and iNOS transcription while mRNA levels of the immunosuppressive cytokines IL-10 and TGF- $\beta$ were reduced by both BCG strains (Fig. 1b). We confirmed these patterns of cytokine expression at the protein level using ELISA for TNF- $\alpha$, IL-2, and IFN- $\gamma$, and noted that intravesical BCG-disA-OE, strongly increased the levels of IFN- $\gamma$ in rat spleens while BCG-WT did not (Fig. S2a). Correspondingly, we found a significant decrease in highest pathology grade (Fig. 1c), tumor involvement index (Fig. 1d) and highest tumor stage (Fig. 1e) in rats treated with BCG-disA-OE in comparison to untreated. By tumor involvement index BCG-disA-OE was statistically significantly superior to no treatment $(p<0.001)$ and to BCG-WT $(p<0.05)$, whereas BCG-WT showed only a trend towards improvement over no treatment. Importantly, the highest tumor stage observed in BCG-disA-OEtreated rats was CIS, whereas it was T1 in those receiving BCG-WT, and T2 in untreated rats, and $53.3 \%$ of BCG-disA-OE-treated rats were cancer free $(p=0.009)$ compared with $31.2 \%$ of BCG-WT and $0 \%$ of the untreated rats (Fig. 1e). Immunohistochemical analyses revealed a significant reduction in Ki67 staining in BCG-disA-OE-treated MNU rat bladders when compared to untreated $(p<0.01)$ and BCG-WT $(p<0.05)$ suggesting reduced tumor proliferation (Fig. 1f). CD68 staining of rat bladder showed significantly higher levels of macrophage recruitment, and a significant reduction in CD206 staining in the BCG-disA-OE-treated MNU-rats compared to those receiving BCG-WT (Fig. 1g). Among macrophages present, there was a trend toward elevation of the pro-inflammatory M1-like marker CD86 (Fig. 1g) and significant reduction in CD206 staining, an M2-like marker associated with resolution of inflammation, in the BCG-disA-OEtreated rats compared with untreated controls. These observations indicate that the enhanced induction of type I IFN and other proinflammatory signatures in bladders of tumor-bearing rats 
treated with BCG-disA-OE correlated with the enhanced antitumor activity of the recombinant BCG strain.

To validate our findings in a second cancer model, we tested the efficacy of BCG-disAOE against bladder cancer cells derived from mice in a syngeneic MB49 model of urothelial cancer. Following flank engraftment with MB49 tumor cells, mice received four intratumoral treatments over 9 days as shown in Fig. 1h. In this model we observed a significant reduction in MB49 tumor volume and tumor weight after intratumoral injection of BCG-disA-OE when compared with BCG-WT (Fig. 1i and Fig. S2b). Histopathology demonstrated extensive necrosis and congestion in MB49 tumors treated with BCG-disA-OE when compared to BCG-WT and untreated (Fig. S2c). There were no significant changes in body weights of mice receiving BCG, however spleen weight of MB49 tumor mice significantly increased (Fig. S2d) after intratumoral BCG injection. We further characterized the impact of the treatments on macrophage polarization and recruitment of activated T cells in the tumor microenvironment (TME). As shown in Fig. 1j, compared with BCG-WT, BCG-disA-OE significantly reduced the abundance of immunosuppressive M2 macrophages when compared to untreated and BCG-WT and significantly $(p<0.01)$ increased proinflammatory M1 macrophages. Similarly, BCG-disA-OE recruited significantly more IFN- $\gamma$-producing $\mathrm{CD}^{+} \mathrm{T}$ cells when compared to BCG-WT, and both BCG strains increased IFN- $\gamma$-producing $\mathrm{CD}^{+} \mathrm{T}$ cells. While both BCG strains recruited more $\mathrm{CD}^{+}$and $\mathrm{CD} 8^{+}$cells to the tumors, BCG-disA-OE uniquely increased $\mathrm{CD} 8^{+} \mathrm{T}$ cells in the spleens of treated animals (Fig. S3a). BCG-disA-OE also significantly reduced tumor-associated Tregulatory (Treg) cells to a greater degree than BCG-WT in both tumor and spleen (Fig. S3b). These results indicate that in this murine model of urothelial cancer, BCG-disA-OE has superior antitumor efficacy than BCG-WT, and its efficacy correlates with shift in polarization of macrophages to $\mathrm{M} 1$, increased activation of both $\mathrm{CD} 4^{+}$and $\mathrm{CD} 8^{+} \mathrm{T}$ cells, and a reduction of local intratumoral and systemic Treg cell populations. 


\section{BCG-disA-OE is a potent inducer of STING signaling and proinflammatory cytokine} secretion by macrophages in vitro

BCG is known to be internalized by macrophages and other phagocytic cells ${ }^{30}$, and mycobacteria have been shown to release c-di-AMP into the cytosolic compartment where it activates the STING pathway (Fig. 2a) ${ }^{18,26}$. To further investigate the mechanisms of antitumor activity of BCG-disA-OE, we evaluated its immunologic effects on human monocyte-derived macrophages (HMDMs), primary murine bone marrow-derived macrophages (BMDM), and dendritic cells (BMDC) as well as macrophage cell line (J774.1). We found consistent induction of IRF3, IFN- $\beta$, IFN- $-\gamma$, TNF- $\alpha$ and IL-6 in all cell types in response to BCG-disA-OE that was significantly higher than that seen with BCG-WT-exposed cells (Fig. 2b-d and Fig S4a-c), and in human MDM and murine BMDM this difference was accentuated by IFN- $\gamma$ treatment (Fig. S4de). These differences were strictly STING-dependent as confirmed using BMDM from STING ${ }^{-/}$ mice (Fig. 2c). Since STING activation also leads to upregulation of NF-кB via the TBK1 pathway, we found that expression of both TNF- $\alpha$ and IL- 6 in the same panel of cells paralleled that of IFN$\beta$ and was significantly higher following exposure to BCG-disA-OE compared with BCG-WT (Fig. 2d and S4c). STING agonists are known to be potent inducers of several chemokines (CXCL9, CXCL10 [IP-10], CXCL22, and MCP-1) as well as iNOS ${ }^{31,32}$ and consistent with this, IFN- $\gamma$ activated BMDMs showed a more robust induction of these chemokines and iNOS when challenged with BCG-disA-OE strain than with BCG-WT (Fig. 2e) as we also observed in vivo in the MNU-rat model of NMIBC (Fig. 1b). We also assessed the cellular toxicity using annexinPI staining and found that whereas late apoptotic cell death remained at baseline with BCG-disAOE exposure in both BMDM and J774.1 macrophage, BCG-WT exposure elicited significantly higher levels of apoptotic cell death (Fig. S5a-b) in the BMDM cells. These observations demonstrate the BCG-disA-OE elicits pro-inflammatory cytokine expression more potently than 
BCG-WT in primary human MDM as well as murine primary macrophages and macrophage cell lines.

\section{BCG-disA-OE is also a strong inducer of pro-inflammatory cytokines in urothelial} carcinoma cells in vitro.

Because urothelial cells are capable of BCG phagocytosis and cytokine secretion ${ }^{33-35}$, we sought to determine if the STING agonist-overexpressing BCG strain elicits altered effects in urothelial carcinoma cells in vitro. Using monolayer cultures of 5637 (a human urothelial cancer cell line), we confirmed rapid BCG internalization of both BCG-WT and BCG-disA-OE as early as 30 mins after exposure (Fig. S6a) and documented that tumor cell survival was similar following BCG exposure with different MOI levels in three separate human urothelial cancer cell lines (Fig. S6b). Next, we tested a panel of human and rodent urothelial carcinoma cell lines representing various tumor stages and observed that BCG-disA-OE elicited more potent gene and protein expression of the pro-inflammatory cytokines TNF- $\alpha$, IL-6, IL 1- $\beta$ than BCG-WT in all the cell lines tested (Fig. 3a-d and Fig. S7a-b). In light of the fact that increased cytokine signaling from STING activation elicits increased autophagy ${ }^{18,36,37}$ we evaluated the formation of LC3B puncta in human 5637 urothelial cancer cells exposed to BCG-disA-OE and BCG-WT. The majority of BCG-disAOE bacilli co-localized with LC3B suggesting enhanced autophagic targeting by BCG-disA-OE, while similar co-localization was not observed with BCG-WT (Fig. 3e). These observations are consistent with the concept that urothelial cells contribute to immune activation following BCG exposure and demonstrate that they display increased pro-inflammatory signaling by BCG-disAOE in agreement with enhanced efficacy we observed in vivo.

\section{BCG-disA-OE-exposed macrophages are classically activated and inflammatory in nature.}

The immunotherapeutic efficacy of BCG has been correlated with increased levels of inflammatory or M1-phenotype macrophages in $\mathrm{NMIBC}^{38}$. Having observed an increased 
abundance of M1-like macrophages in MNU rat bladders following BCG-disA-OE instillation (Fig. 1g-h) and higher levels of several M1-associated cytokines and chemokines in macrophages exposed to BCG-disA-OE (Fig. 2d-e), we next measured the expression of immune markers delineating the M1 versus M2 macrophage phenotypes in the presence of BCG-WT or BCG-disA$\mathrm{OE}$ in both murine and human primary macrophages. First, we focused on the MHC class IIexpressing $\mathrm{CD} 45^{+} \mathrm{CD} 11 \mathrm{~b}^{+} \mathrm{F} 4 / 80^{+}$murine BMDM population following in vitro BCG exposure (Fig. S8a-b). As may be seen in Fig. 4a and Fig. S9a-b, we observed a significant expansion of TNF$\alpha$-expressing $\mathrm{CD} 11 \mathrm{~b}^{+} \mathrm{F} 4 / 80^{+}$murine BMDMs following exposure to BCG-disA-OE compared with BCG-WT. We next gated on cells expressing the M2 surface receptors CD206 ${ }^{+}{\text {CD } 124^{+}}^{+}$ (frequently reported as immunosuppressive of anti-tumor immunity) among CD45 ${ }^{+} \mathrm{CD} 11 \mathrm{~b}^{+} \mathrm{F} 4 / 80^{+}$ macrophages and observed a reduction of this population with BCG-disA-OE relative to BCG-WT (Fig. 4a and Fig. S9c-d). Within this immunosuppressive cell population, there was a high proportion of IL-10-expressing CD206 ${ }^{+}$CD $124^{+}$cells in BCG-WT-exposed macrophages, while IL-10-expressing cells were significantly reduced in response to BCG-disA-OE exposure (Fig. 4a and Fig. S9e-f). These results demonstrate that compared with BCG-WT, BCG-disA-OE exposure elicits more extensive macrophage reprogramming with expansion of pro-inflammatory M1 macrophages displaying increased antigen presentation (MHC class II expression) and TNF$\alpha$ expression and contraction of immunosuppressive M2 macrophages expressing IL-10.

Myeloid-derived suppressor cells (MDSCs) are a heterogeneous population of immature myeloid cells known to foster immunosuppression within the $\mathrm{TME}^{39,40}$. Accordingly, we investigated the induction of monocytic-myeloid derived suppressor cells, M-MDSCs, (CD45 Ly6C $^{\text {hi }}$ Ly6G $^{-}$CD11 $\mathrm{b}^{+} \mathrm{F} 4 / 80^{-}$) using primary murine BMDMs (Fig. S10). We observed a significant expansion of M-MDSCs following BCG-WT exposure, while this same population was significantly smaller following BCG-disA-OE exposure (Fig. 4a and Fig. S11a-b). Moreover, the M-MDSCs elicited by BCG-WT exhibited higher IL-10 expression, whereas IL-10-expressing M-MDSCs were 
virtually absent after BCG-disA-OE exposure (Fig. 4a and Fig. S11c-d). These observations suggest that BCG may contribute to an enhanced immunosuppressive TME (potentially contributing to BCG-unresponsiveness in some NMIBC patients) that may be overcome with the BCG strain overexpressing a STING agonist.

We next characterized the macrophage activation phenotypes in HMDMs isolated from several independent healthy human donors. Both the WT and BCG-disA-OE strains elicited increases in the population of classical macrophages (CD11b $\left.{ }^{+} \mathrm{CD} 14^{+} \mathrm{CD}^{-} 6^{-}\right)$, but these inductions were comparatively higher in response to BCG-disA-OE (Fig. 4b and Fig. S12a-c). We considered antigen-presenting classically activated macrophages (CD14 ${ }^{+}$CD16 $^{-} \mathrm{HLA}^{-\mathrm{DR}^{+}}$) and their ability to produce TNF- $\alpha$ or IL-6 and found a significantly increased proportion of TNF$\alpha$ and IL6- producing HLA-DR ${ }^{+}$cells following exposure to BCG-disA-OE compared to BCG-WT (Fig. 4b and Fig. S13a-b). We also investigated the immunosuppressive M2 surface markers, $\mathrm{CD}^{2} 6^{+}$and $\mathrm{CD} 163^{+}$, on transitional or intermediate macrophages $\left(\mathrm{CD} 11 \mathrm{~b}^{+} \mathrm{CD} 14^{+} \mathrm{CD} 16^{+}\right)$and found a consistent decrease in them following BCG-disA-OE exposure (Fig. 4c and Fig. S12b and Fig. 13b). Consistent with our findings in murine macrophages, the fraction of these immunosuppressive, intermediate macrophages expressing M2 surface markers and IL-10 was also significantly lower in response to exposure to BCG-disA-OE than with BCG-WT (Fig. 4c and Fig. S13b). In summary, using both mouse and human primary macrophage ex vivo models, we found that, compared with BCG-WT, BCG-disA-OE promotes greater macrophage activation towards an M1 phenotype (inflammatory), and concomitantly reduces the emergence of cells with immunosuppressive markers, including M-MDSCs.

\section{Macrophages harboring BCG-disA-OE are highly phagocytic.}

Macrophages exposed to STING agonists delivered by intratumoral injection have been reported to display increased phagocytosis and other markers of macrophage activation; they 
also recruit new macrophages which serve a phagocytic role in the $\mathrm{TME}^{41-44}$. Consistent with these observations we confirmed that HMDMs transfected with c-di-AMP showed increased phagocytosis and exhibited elongated dendrites compared to mock-transfected populations (Fig. S14). We then evaluated the phagocytic properties of HMDMs following exposure to the different BCG strains and found significantly greater phagocytosis of IgG-opsonized FITC-latex beads by macrophages harboring BCG-disA-OE strains compared to those harboring BCG-WT (Fig. 4d). In keeping with the previously established role of STING activation in augmenting autophagy ${ }^{18,36,37}$, we found that a majority of intracellular BCG-disA-OE bacilli were co-localized with LC3B in IFN- $\gamma$-activated primary BMDMs (Fig. 4e-f), similar to our findings with urothelial cancer cells (Fig. 3d), while autophagy induction in BCG-WT was significantly lower. We also found significantly greater co-localization of BCG-disA-OE bacilli with the autophagy adapter protein p62 compared to that observed with BCG-WT (Fig. 4g-h). These results reveal BCG-disAOE increases the levels of phagocytosis and autophagic processing within macrophages to a greater degree than BCG-WT, a phenomenon associated with enhanced peptide antigen presentation to $\mathrm{MHC}$ class-II molecules ${ }^{45,46}$.

5/8/20 7:07:00 PM

\section{BCG-disA-OE reprograms macrophages epigenetically and potentiates trained immunity.}

In light of recent data showing BCG to be a potent inducer of long-lived innate immunity termed trained immunity - through epigenetic modifications of key pro-inflammatory genes ${ }^{10,11,13}$ we hypothesized that the addition of STING agonist overexpression to standard BCG might potentiate the epigenetic hallmarks of trained immunity in primary human monocytes. Having already established that BCG-disA-OE is a more potent inducer of macrophage TNF- $\alpha$ and IL-6 secretion than BCG-WT, we confirmed this in primary human monocytes from a group of 6 healthy human subjects (Fig. 5a). The ability of traditional BCG to elicit trained immunity has been correlated with changes in epigenetic marks that increase pro-inflammatory gene expression ${ }^{12}$. 
Thus, we asked if the enhanced induction of TNF- $\alpha$ and IL-6 expression elicited by BCG-disA-OE compared with BCG-WT is epigenetically mediated. To this end, we evaluated the promoter regions of the TNF- $\alpha$ and IL-6 genes for durable, antigen-independent epigenetic changes using an assay ${ }^{47}$ in which human monocytes exposed to BCG strains for $24 \mathrm{~h}$ were rested for five days prior to challenge with a heterologous PAMP (pathogen-associated molecular pattern), the TLR1/2 agonist Pam3CSK4 on day 6 (Fig. 5b). Using chromatin immunoprecipitationpolymerase chain reaction (ChIP-PCR) assays, we quantified the activating histone methylation mark H3K4me3 present in the TNF- $\alpha$ and IL-6 promoters. We observed that exposure to BCGdisA-OE led to greater enrichment of this mark than BCG-WT even without the heterologous second stimulation (i.e., adding RPMI media alone at day 6). Upon re-stimulation with Pam3CSK4 at day 6 , the abundance of the activating epigenetic mark was further increased by both BCG strains, but BCG-disA-OE-pretreatment yielded notably more enrichment than BCG-WT (Fig. 5c). Similarly, we investigated the chromatin repression mark $\mathrm{H} 3 \mathrm{~K} 9 \mathrm{me} 3$ at the same two promoters and found that, while both BCG strains led to reduced levels of H3K9me3 (which were further accentuated by addition of Pam3CSK4), the degree of reduction mediated by BCG-disA-OE was consistently greater than that mediated by BCG-WT, both upon initial exposure and after rest and re-stimulation (Fig. 5d). Simultaneous measurement of TNF- $\alpha$ and IL-6 in BCG-trained culture supernatant following non-specific stimulation by Pam3CSK4 revealed that BCG-disA-OE-trained macrophages produced significantly higher levels of these pro-inflammatory cytokines than did those trained with WT-BCG (Fig. 5e-f). Next, an LC-MS based investigation of the immunometabolic state of primary human and murine macrophages exposed to BCG-disA-OE suggested increased intracellular glucose and lactate as compared to BCG-WT-exposed macrophages (Fig. 5g-i), suggesting an enhanced glycolytic state in macrophages infected with BCG-disA-OE. In the same screen, there was a trend towards increased levels of intracellular, tryptophan and significantly lower levels of immunosuppressive kynurenine (Fig. $\mathbf{5 g}$-i) suggesting 
more extensive metabolic reprograming in the BCG-disA-OE-infected macrophages compared with those infected with WT-BCG. In addition, modulation of several key metabolites such as itaconate and UDP-N-acetyl-glucosamine (UDP-GlcNac) suggest extensive and stronger metabolic shift in BCG-disA-OE macrophages. Together, these results reveal that overexpression of a STING agonist by BCG potentiates the degree of epigenetic reprogramming normally conferred by BCG-WT and that in turn leads to increased proinflammatory cytokine responses and immunometabolic changes associated with improved antitumor immunity in the TME ${ }^{48-50}$.

\section{BCG-disA-OE is less pathogenic than BCG-WT in two mouse models.}

To assess the pathogenicity of the two BCG strains, we used an immunocompetent $\mathrm{BALB} / \mathrm{c}$ mouse model of aerosol exposure and measured the lung bacillary burden after four weeks when adaptive immune responses are maximal (Fig. 6a). While the day 1 implantation of the two BCG strains was equivalent, we observed that BCG-disA-OE proliferated in murine lungs to a significantly lower degree than BCG-WT by a margin of $0.43 \log _{10}$ colony forming units (Fig. 6b and Fig. S15a). As we observed in earlier in cell-based models, pro-inflammatory cytokine levels in both lungs and spleens were significantly higher in BCG-disA-OE-exposed mice than those receiving BCG-WT (Fig. 6c-d and Fig. S15b). We also tested the two strains in immunocompromised SCID mice, again using a low dose aerosol exposure model (Fig. 6e) and observed a statistically significant survival prolongation with BCG-disA-OE compared to BCG-WT (Fig. 6f and Fig. S15c). Thus, despite eliciting more profound inflammatory signatures in numerous model systems, BCG-disA-OE is less pathogenic than BCG-WT in these two murine model systems.

\section{DISCUSSION}

Bladder cancer ranks as the ninth most common cancer worldwide, and approximately $75 \%$ of patients diagnosed with bladder cancer present initially with NMIBC ${ }^{51}$. For over 40 years, 
transurethral resection of the bladder mass followed by BCG adjuvant immunotherapy has been the standard of care for high-risk NMIBC. Although BCG has been shown to reduce tumor recurrence and progression, $\sim 30-50 \%$ of patients will recur after BCG, placing them at a high risk of recurrence and progression to $\mathrm{MIBC}^{52,53}$. This high risk for recurrence combined with the lack of effective alternative or salvage therapies, and a worldwide shortage of BCG has generated an unmet need for improved versions of $\mathrm{BCG}^{1,2}$. The economic burden and inability to develop effective bladder-sparing approaches in BCG-unresponsive and relapsing populations prompted us to investigate alternative mechanism-based therapies exploiting the STING-signaling pathway ${ }^{54}$.

A primary goal of this study was to determine whether the antitumor efficacy of BCG in urothelial cancer models could be enhanced by engineering excess production of the STING agonist, c-di-AMP. In light of studies showing elevated Type I IFN and NF-KB-mediated antitumor host immune responses in NMIBC patients who respond to $\mathrm{BCG}^{55-57}$, we reasoned that engineering STING agonist overexpression into traditional BCG might further increase these favorable immune parameters. c-di-AMP is a STING agonist produced naturally in low levels by BCG (where it serves as a second messenger signaling molecule in microbial physiology), that is closely related to the natural human STING ligand, CGAMP ${ }^{58,59}$. In a rat model of NMIBC, we found that whereas invasive tumors developed in untreated tumor-bearing rats (highest tumor grade of T2) as well as BCG-WT-treated animals (highest tumor grade of T1), invasive bladder cancer was completely absent in rats treated with BCG-disA-OE. Similarly, in the MB49 mouse model of bladder cancer, BCG-disA-OE was superior to BCG-WT in reducing tumor growth with associated increase in tumor necrosis, and these effects were accompanied by significantly higher recruitment of $\mathrm{M} 1$ macrophages, infiltrating IFN- $\gamma$-producing CD4 cells, and reduced accumulation of Treg cells in the tumors. In addition to observing improved antitumor efficacy, we found that BCG-disA-OE was less pathogenic than BCG-WT in two mouse models, suggesting 
that BCG-disA-OE may be safer than BCG-WT and that STING agonist overexpression may not result in an undesirable adverse event profile.

BCG vaccination for tuberculosis has long been known to confer heterologous crossprotective effects against antigenically unrelated viral and bacterial infections ${ }^{10,11,60}$. This phenomenon has recently been shown to be mediated by an innate immune mechanism called trained immunity in which epigenetic modifications following BCG exposure confer an elevated set-point of transcriptional activation in genes governing pro-inflammatory responses including cytokine genes, immunometabolism and cell polarization ${ }^{12,61-64}$. Trained myeloid cells demonstrate elevated responses to subsequent challenge by unrelated antigens or pathogens, and the effect is long-lasting since transcriptional changes are induced by BCG in hematopoietic stem cells and myeloid progenitor cells ${ }^{11}$. These same trained immunity changes elicited by BCG may underlie the immunotherapeutic effects of BCG in cancer prevention ${ }^{65-68}$. Therefore, another goal of this study was to evaluate whether or not the salutary effects of BCG in bladder cancer therapy are mediated through a trained immunity mechanism, and how overexpression of a STING agonist may modulate BCG-mediated trained immunity.

Indeed, we observed that BCG-disA-OE potentiated trained immune responses to a greater degree than BCG-WT in multiple in vivo and in vitro analyses. We observed elevated levels of pro-inflammatory cytokines and chemokines in bladders from tumor-bearing animals treated with BCG-disA-OE compared to BCG-WT. Since non-immune cells have also been shown to possess immunological memory ${ }^{69}$, we considered the possibility that this cytokine response may have originated from myeloid cells in the TME and/or the tumor cells themselves. Indeed, we found that compared with BCG-WT, BCG-disA-OE elicited more potent cytokine responses in both primary macrophages and urothelial cancer cells representing various tumor stages. We found dramatically reduced induction of these cytokines in BMDMs from STING ${ }^{-/-}$mice, indicating for the first time that that this trained immunity is a downstream consequence of STING activation. 
In addition, we found robust induction of several chemokines as has been observed in other studies with stimulation using exogenous STING agonists ${ }^{22,32}$.

Enhanced trained immune responses leading to reacquisition of pro-inflammatory traits of macrophages, also called repolarization, has been shown to correlate with increased survival in tumor-bearing mice and in cancer patients ${ }^{44,70}$. Consistent with this we observed that, compared with BCG-WT, BCG-disA-OE led to greater increases in M1 macrophages in vivo in two separate in vivo models of urothelial cancer, and we noted the same pattern in vitro with both mouse and human primary macrophages. STING activation in host cells is known to augment autophagy, and polymorphisms in autophagy-related genes correlate with progression and recurrence of bladder cancer following BCG treatment ${ }^{71}$. Consistent with this, we observed that BCG-disA-OEtreated myeloid cells also exhibited enhanced phagocytosis and autophagy to a greater degree than BCG-WT. This finding raises the intriguing possibility that tumor-associated macrophages that take up BCG or BCG-disA-OE may also augment adaptive immunity through enhanced antigen presentation of tumor-associated peptides to the lymphocytes.

MDSCs promote tumor growth and progression by suppressing host $\mathrm{T}$ cell immune responses and are commonly elevated in many tumors, including bladder cancers ${ }^{39,72,73}$. Interestingly, we observed that treatment of murine macrophages with BCG-WT in fact induced a higher percentage of M-MDSCs compared with untreated controls, while M-MDSCs were essentially absent in BCG-disA-OE treated macrophages. These findings suggest that classical (WT) BCG may promote some degree of immunosuppression in the TME that may partly offset the benefits of a pro-inflammatory M1 repolarization. This BCG-mediated expansion of M-MDSCs may contribute to BCG unresponsiveness in certain NMIBC patients, and it may explain the observation that in countries which routinely use BCG for TB prevention, vaccinees display reduced levels of asthma and atopic dermatitis ${ }^{74,75}$. In contrast, expansion of M-MDSCs in macrophages by classical (WT) BCG was reversed by STING agonist overexpression which is in 
keeping with a recently unveiled function of the STING-SOCS1-STAT3 pathway in promoting antitumor immunity by down-regulating the induction of MDSCs in nasopharyngeal carcinoma ${ }^{76}$.

Trained immunity induced in macrophages is mediated by epigenetic reprogramming at the level of histone methylation and acetylation and is associated with elevated expression of genes involved in glucose metabolism ${ }^{12}$. We found a significant enrichment of the activating H3K4me3 mark on the TNF- $\alpha$ and IL-6 gene promoters in BCG-disA-OE-trained human monocytes as compared to those trained by BCG-WT and a concomitant reduction of the H3K9me3 repression mark. In accordance with the epigenetic changes, BCG-disA-OE-trained macrophages released more TNF- $\alpha$ and IL-6 following TLR1/2 engagement as compared to macrophages trained with WT-BCG. Similarly, we observed that compared with BCG-WT, BCGdisA-OE led to an increased level of lactate and glycolytic pathway substrates as well as reduced tryptophan conversion to kynurenine--metabolomic signatures consistent with trained immunity.

In summary, our findings reveal a significant enhancement of BCG's antitumor activity with a reengineered BCG that overexpresses a STING agonist. Importantly, STING pathway activation potentiates the expression of pro-inflammatory cytokines and chemokines and induces macrophage polarization towards an M1 phenotype. We also found that BCG-disA-OE elicits epigenetic changes that govern cytokine expression which are known to be mechanistically linked to immunometabolic and cell polarization changes. These observations reveal that boosting STING agonism improves the ability of BCG to elicit trained immunity and also its immunotherapeutic benefit against NMIBC. This association strongly suggests that a fundamental mechanism of BCG's efficacy in urothelial cancer is through trained immunity. 


\section{Methods: Online Methods}

Bacterial strains and culture conditions: In this study we used Mycobacterium bovis (M. bovis) Bacillus Calmette- Guérin (BCG) Pasteur (BCG-WT Pasteur) (a generous gift from Dr. Frank Collins [FDA] and identical to BCG-Pasteur provided by the Pasteur Institute to the Trudeau Institute in 1967 as TMC No. 1011) and commercially available BCG-Tice (Onco-Tice ${ }^{\odot}$, Merck) for for generation of c-di-AMP overexpressing recombinant BCG strains. Briefly, genomic DNA from Mycobacterium tuberculosis (M. tb) strain CDC1551 was used for PCR amplification of disA (MT3692/Rv3586). Single isolated bacterial colonies growing on 7H11 plates supplemented with oleic-albumin-dextrose-catalase (OADC) (Cat. B11886, Fisher Scientific) were picked and propagated in 7H9 Middlebrook liquid medium (Cat. B271310, Fisher Scientific) supplemented with (OADC) (Cat. B11886, Fisher Scientific), 0.5\% glycerol (Cat. G55116, Sigma) and 0.05\% Tween-80 (Cat. BP338, Fisher Scientific). Cloning experiments were performed using E. coli strain DH5- $\alpha$ (Cat. 18258012, Fisher Scientific) and was routinely maintained in LB broth. For generation of disA overexpressing BCG, an E. coli-mycobacterial shuttle vector (pSD5.hsp60) was used to clone M.tb gene MT3692 or Rv3586 under the strong mycobacterial promoter hsp60 as described earlier ${ }^{18}$. Clones were confirmed by gene sequencing and were used for bacterial transformation by electroporation method. Recombinant strains were confirmed using colony PCR against kanamycin cassette, subjected to whole genome sequencing and qPCR analyses. Details of all bacterial strains, plasmids and constructs are listed in supplementary table S1.

\section{Mammalian cell culture:}

Cell lines: For cell-based in vitro infection assays J774.1 (American Type Culture CollectionATCC $®$ TIB67 ${ }^{\mathrm{TM}}$, Manassas, VA, USA) murine macrophage cell lines were cultivated in RPMIGlutamax (Cat. 61870-036, Fischer Scientific), supplemented with 10\% heat inactivated fetal 
bovine serum (FBS) (Cat. 10082147, Fischer Scientific) with $1 \%$ streptomycin/penicillin at $37^{\circ} \mathrm{C}$ with $5 \% \mathrm{CO}_{2}$. Urothelial carcinoma cell lines 5637 (ATCC® HTB-9 ${ }^{\mathrm{TM}}$ ), a human high grade urothelial cancer; RT4 (ATCC $\left.{ }^{\circledR} \mathrm{HTB}^{\mathrm{TM}}\right)$, a human transitional cell low grade urothelial cancer; J82 (ATCC $®$ HTB-1 $\left.{ }^{\mathrm{TM}}\right)$, a human high grade urothelial cancer; and NBT II (ATCC $® \mathrm{CRL}-1655^{\mathrm{TM}}$ ), N-butyl-N-(4-hydroxybutyl) nitrosamine induced tumor cell line in Rattus norvegicus Nara Bladder Tumor No. 2, UPPL1595 (luminal cell line established from a spontaneous primary bladder tumor in an Uroplakin-Cre driven PTEN/P53 knockout genetically engineered mouse model and were generously provided by Dr. William Kim (UNC Chapel Hill)., BBN975 (basal- cell line established from , 0.05\% N-Butyl-N-(4-hydroxybutyl) nitrosamine (BBN) induced murine urothelial cancer model and was generously provided by Dr. William Kim (UNC Chapel Hill), and MB49 (murine urothelial carcinoma cells, 7,12-dimethylbenz[a]anthracene (DMBA, EMD Millipore, Cat. SSC148) were maintained as monolayer in RPMI 1640 medium supplemented with $10 \%$ heat inactivated fetal bovine serum (FBS) with $1 \%$ streptomycin/penicillin at $37^{\circ} \mathrm{C}$ with $5 \% \mathrm{CO}_{2}$. Mouse fibroblast cell line NCTC clone 929 [L cell, L-929, derivative of Strain L] (ATCC $®$ CCL-1 ${ }^{\mathrm{TM}}$ ) were routinely maintained as monolayer in DMEM media supplemented with $10 \%$ heat inactivated fetal bovine serum (FBS) with $1 \%$ streptomycin/penicillin at $37^{\circ} \mathrm{C}$ with $5 \% \mathrm{CO}_{2}$. All cell lines were not maintained more than 10 passage cycle and Mycoplasma testing was performed periodically while cells were in culture. Reporter mouse cell line, RAW-Lucia ISG (InvivoGen, CA, USA) was cultivated in custom prepared media as per manufacturer's instructions.

Primary cells (Macrophages and Dendritic Cells): For generation of murine bone-marrowderived macrophages (BMDMs) and dendritic cells (BMDCs), bone marrow (BM) cells were isolated from 4-week old wild-type (WT) C57BL/6J (Charles River laboratories, North Wilmington, Mass) and STING-KO mice (C57BL/6J-Tmem173gt/J, Jackson laboratories). Multiple vials of bone-marrow cells were preserved in cryopreservation media containing 10\% DMSO (Cat. D2650; Sigma) and 90\% heat inactivated FBS (Cat. 10082147, Fischer Scientific) in liquid 
nitrogen. For differentiation of BM cells into macrophages or DCs, random cryopreserved vials were chosen and differentiated for 6 days in BMDM-differentiation media made from DMEM containing 10\% FBS, 1\% MEM amino acids (Cat. 11130051, Thermo Fisher Scientific), 1\% MEM non-essential amino acids (Cat. 11140050, Thermo Fisher Scientific), 1\% sodium pyruvate (Cat. 11360070, Thermo Fisher Scientific), 1\% MEM vitamin (Cat. 11120052, Thermo Fisher Scientific) and antibiotics (Penicillin-Streptomycin solution) supplemented with $30 \%$ sterile mouse fibroblast L929 (ATCC $®$ CCL-1 ${ }^{\mathrm{TM}}$ ) conditioned media. Differentiation of BM cells into DCs was carried out in low attachment $10 \mathrm{~mm}$ cell culture dish in presence of bone marrow-differentiation media in presence of recombinant murine Granulocyte-Macrophage Colony-Stimulating Factor (GM-CSF) (Cat. 315-03, Peprotech) for 48 h. Non-adherent cells were washed and loosely attached cells were allowed to differentiate into BMDCs for next 6 days. Cells were characterized for macrophage and DC markers using cell-surface staining and flow cytometry analyses. Human primary monocytes and human monocyte-derived macrophages (HMDMs) were used for cellbased in viro infection assays. Peripheral blood-derived mononuclear cells (PBMCs) isolated from healthy male donors (leukopacks) aged between 18-30 were used for isolation of human monocytes (HM) or human monocyte-derived macrophages (HMDM). Briefly, to separate blood constituents and isolation of buffy coat density gradient centrifugation $\left(400 \times g\right.$ at $18^{\circ} \mathrm{C}$ for $\left.30 \mathrm{~min}\right)$ of RPMI-1640 diluted blood over a Ficoll-Paque ${ }^{\text {TM }}$ Plus reagent (Cat. 17-1440-02, GE Healthcare, Piscataway, NJ) was performed. Cells were washed several times using $1 \times$ PBS and were counted using hemocytometer. Once counted $\mathrm{CD} 14^{+}$human monocytes were isolated from PBMCs using magnetic labeling (Monocyte Isolation Kit II, Cat. 130-091-153, Miltenyi Biotec, San Diego, CA) and magnetic columns as per manufacturer's instructions. The purity of isolated CD14 ${ }^{+}$cells was confirmed using a fraction of cells stained with a fluorochrome-conjugated antibody against a monocyte marker as recommended by manufacturer and cells were analyzed using BD-LSR2 flow cytometer. Human monocytes were seeded $\left(2.0-3.0 \times 10^{5}\right.$ cells / ml in RPMI 1640 medium supplemented with $10 \% \mathrm{FBS}$ and $1 \%$ streptomycin/penicillin at $37^{\circ} \mathrm{C}$ with $5 \% \mathrm{CO}_{2}$. 
Monolayers of CD14+ monocytes were differentiated into M1 [GM-CSF (20 ng/ml, PeproTech, Rocky Hill, NJ) and IFN- $\gamma(20$ ng/ml, PeproTech, Rocky Hill, NJ PeproTech)] or M2 [M-CSF (20 ng/ml, PeproTech, Rocky Hill, NJ) and IL-4 (20 ng/ml, PeproTech, Rocky Hill, NJ PeproTech)] for next 7 days ${ }^{77}$.

Animals: Experimental procedures involving live animals were carried out in agreement with the protocols approved by the Institutional Animal Care and Use Committee (IACUC) at The Johns Hopkins University School of Medicine. For animal infection protocols, pathogen-free age 4-6 weeks female C57BL/6J (Charles River Laboratories, North Wilmington, Mass) and Fox Chase SCID mice (Charles River Laboratories North Wilmington, Mass.) were purchased and housed under pathogen-free conditions at an Animal Biosafety Level-3 animal facility without crossventilation. Fischer 344 female rats age 8 weeks (Harlan, avg. weight 160g) were housed at an BSL2 animal facility. Animals were given free access to water and standard chow and were monitored daily for general behavior and appearance by veterinary specialists.

In vitro infection assays: For in vitro infection assays, cell lines or primary cells were seeded at required cell density in 6-well tissue culture plates or $10 \mathrm{~mm}$ petri dishes. For infection, log-phase wild-type and BCG-disA-OE strains were harvested by centrifugation and washed twice using DPBS to remove residual detergent and BSA then suspended in antibiotic-free RPMI 1640 media supplemented with $10 \%$ FBS. For infection assays, the bacteria were deposited at pre-calibrated multiplicity of infection (MOI). Infection was allowed for next 4 hours, followed by repeated washing of infected cells using warm DPBS to remove non-internalized bacteria. Infected cells were incubated until endpoints in presence of RPMI-1640 medium supplemented with 10\% FBS and antibiotics. 
Toxicity assays: Human urothelial cancer cell lines, RT4, 5637 , and J82, were cultured at $37^{\circ} \mathrm{C}$ under $5 \% \mathrm{CO}_{2}$ in RPMI 1640 containing 10\% FBS without antibiotics. For cell toxicity assay, 3000 cells for RT4 and 1500 cells for 5637 and J82 were seeded in a 96-well tissue-treated plate in triplicate, respectively. Twenty-four hours after seeding, cells were treated with the indicated ratio of BCG to cells for 72 hours. To measure cell viability, CellTiter-Glo Luminescent Cell Viability Assay (Promega, Madison, WI, USA) and FLUOstar OPTIMA (BMG Labtech, Ortenberg, Germany) were used according to manufacturer's protocols. Relative cell viability was calculated by dividing the viability of the indicated ratio by that of a control.

For Annexin-PI staining, 0.5 million J774.1 cell and BMDMs were plated per well in 6-well plates for physical attachment. Cells were exposed at 1:10 MOls for 24 hours using wild-type and BCGdisA-OE strains of Tice and Pasteur to determine the BCG cytotoxicity following exposure. At the endpoint of infection or treatment cells were non-enzymatically removed using $0.02 \%$ EDTA-PBS solution. Cells were washed twice with ice-cold PBS and FITC-annexin-PI was done as per manufacturer's instruction using FITC Annexin V Apoptosis Detection Kit I (Cat. 556547, BD Biosciences). Flow cytometry was performed using a BD LSR II flow cytometer of the Flow Cytometry Core Facility at The Bloomberg School of Public Health, Johns Hopkins University). Data was processed using FlowJo software (Tree Star v10).

Quantitative real-time qPCR: Gene expression profiling was carried out using total RNA isolated from cell lines or primary cells. For RNA isolation from rat bladders, pieces of whole bladder samples were excised, snap frozen in liquid nitrogen immediately after harvesting and stored in RNAlater (Cat. AM7021, Ambion) at $-80^{\circ} \mathrm{C}$. Total RNA isolation was carried out using RNeasy system (Cat. 74106, Qiagen). Real-time qPCR was performed using the StepOnePlus system (Applied Biosystems). For gene expression analyses in cell lines and primary cells, SYBR Fast green double stranded DNA binding dye (Cat. 4085612, Applied Biosystems) was used. Gene expression analyses in rat bladder tissues were performed using TaqMan gene expression 
assays. Gene-specific qPCR primers were purchased from Integrated DNA Technologies and all TaqMan gene expression assays were purchased from Thermo Fischer Scientific. Amplification of RNU6a, $\beta$-actin, GAPDH were used as endogenous control for RNA samples derived from human, mouse and rat cells/tissues respectively. All experiments were performed at least in triplicate and data analyses was done using $2^{-\Delta \Delta C T}$ method. Details of NCBI gene identifiers and primer sequences are given in the supplementary_table S2.

ELISA: Sandwiched ELISA was performed for cytokine (IFN- $\beta$, TNF- $\alpha$, IL-6, IFN- $\gamma$, IL-1 $\beta$ and MCP-1/CCL2) measurement in culture supernatants and animal tissues from lung, spleen or urinary bladder. Briefly, tissues and culture supernatants were flash frozen in liquid nitrogen immediately after harvest and stored at $-80^{\circ} \mathrm{C}$. Animal tissues were homogenized using micro tissue homogenizers (Cat. 1215D61, Kimble) and filter sterilized for measurement of various cytokine protein expression levels using sandwiched ELISA as per manufacturer's recommendations. Details of all ELISA kits and accessory reagents are given in supplementary table S2.

Multicolor confocal microscopy: Multicolor laser confocal microscopy experiments were performed to determine phagocytosis, autophagy, and colocalization studies in urothelial cancer cells and primary macrophages. Briefly, cells were allowed to adhere on sterile glass cover slips placed in 6-well tissue culture plates and infections were carried at pre-calibrated MOI. Log phase bacterial cultures were labeled using FITC (Cat. F7250, Sigma) as described earlier ${ }^{78}$. Following infection and treatment conditions, cells were fixed, permeabilized and blocked followed by overnight incubation with a primary antibody for LC3B (Cat. NB100-2220, Novus) or p62/SQSTM1 (Cat. P0067, Sigma-Aldrich) at recommended dilutions at $4{ }^{\circ} \mathrm{C}$. Cells were washed and incubated in the dark with Alexa Flour 647 conjugated secondary antibody (Cat. A32733, Thermo Fisher Scientific) at $4{ }^{\circ} \mathrm{C}$ for 1 hour. DNA staining was carried out using Hoechst 33342 (Cat. 62249, 
Thermo Fisher Scientific) for 5 minutes. Images were acquired using Zeiss LSM700 single-point, laser scanning confocal microscope at $63 \mathrm{X}$ magnification at the Microscope Facility, Johns Hopkins School of Medicine. Image processing and analyses was carried out using open source Fiji software. For LC3B or p62 quantification, perinuclear LC3B puncta (spot) was counted in a minimum 100 cells across different fields using and Imaris 9.5.0. Quantification carried out using GraphPad Prism software.

Phagocytosis assay: IgG-FITC conjugated latex bead phagocytosis assay kit (Item No. 500290, Cayman Chemicals, USA) was used for phagocytosis studies. Briefly, HMDMs were placed on sterile glass cover slip for attachment. Infection was carried out at 5:1 (HMDM versus BCG) ratio for 3 hours followed by addition of IgG-FITC beads in warm RPMI 1640 media at 1: 400 dilutions for 3 hours. Nuclear staining was carried out using Hoechst 33342 (Cat. 62249, Thermo Scientific) and cells were visualized for bead phagocytosis using Zeiss LSM700 single-point, laser scanning confocal microscope. Quantification of beads was measured by mean fluorescence intensity (M.F.I.) calculations using open source Fiji Software.

Multicolor flow cytometry: The cell surface and intracellular staining was carried out on J774.1, murine BMDMs, human HMDMs and single cells derived from murine MB49 tumors and spleens. Flow cytometry panel were designed and if needed modified form murine myeloid and lymphoid cells and human myeloid cells. Details of all antibodies and the dilutions used are given in the supplementary table S2. For in vitro infection assays, protein transport inhibitor cocktail (Cat. 004980-03, eBioscience) at recommended dilution, 12 hours before harvesting monolayer of cells. At the endpoint cells were harvested using a cell-detachment buffer (ice-cold PBS - 10 mM EDTA solution). Single cell isolation was performed using animal tissues by harvesting tumors and spleens following necropsy. Briefly, tissues were manually disrupted before incubating in collagenase type I (Gibco) and DNase (Roche) in RMPI for 30 minutes at $37^{\circ} \mathrm{C}$. Tumor and 
spleen cells were dissociated through a $70-\mu \mathrm{m}$ filter and washed with PBS. RBC lysis was performed for 5 minutes using ACK lysis buffer (Cat. A1049201, Thermo Fisher Scientific) at room temperature. Cells were washed twice using ice-cold PBS and stained using Zombie Aqua $^{\mathrm{TM}}$ Fixable Viability Kit (Cat. 423101, Biolegend). Cells were washed and resuspended in FACS buffer (1\% BSA, 2mM EDTA in PBS), Fc blocked (TruStain FcX ${ }^{\text {TM }}$, Cat. 101320, and TrueStain Monocyte Blocker ${ }^{\mathrm{TM}}$ Cat. 426102 Biolegend) and stained with conjugated primary antibodies as per manufacturer's protocol. Intracellular staining was performed following fixation and permeabilization (Fixation and Permeabilization Buffer Set, eBioscience). Cells were washed and resuspended in flow buffer and acquired using BD LSRII with FACSDiva Software. analyses were performed using FlowJo (v10) (TreeStar).

The following antibodies were used to stain myeloid and lymphoid cells:

Mouse BMDMs: Anti-CD45 (clone 30-F11), anti-CD124 (clone 1015F8), anti-I-A/I-E (clone 107630), anti-Ly6C (clone HK1.4), anti-CD11b (clone M1/70), anti-F4/80 (clone BM8), anti-Ly6G (clone 1A8), anti CD206 (clone C068C2), anti-TNF (clone MP6-XT22) all Biolegend and anti- IL10 (clone JES5-16E3 eBiosciences).

Human HMDMs: anti CD16 (clone 3G8), anti-CD14 (clone 63D3), anti-HLA-DR (clone L243), antiCD11b (clone ICRF44), anti-CD206 (clone 15-2), anti-CD163 (clone GHI/61), anti-TNF (clone MAb11), and anti-TNF (clone MAb11) all Biolegend.

Mouse macrophages (syngeneic MB49 model of urothelial carcinoma): CD45 (clone 30-F11, Biolegend), CD124 (IL-4Ra) (clone I015F8, Biolegend), I-a/l-e (clone M5/114.15.2, Biolegend), F4/80 (clone BM8, Biolegend), CD206 (clone C068C2, Biolegend), TNF (clone MP6-XT22, Thermo Fisher), IL-10 (clone JES5-16E3, Thermo Fisher)

Mouse T cells (syngeneic MB49 model of urothelial carcinoma): CD45 (clone PerCP, Biolegend), CD25 (clone PC61, Biolegend), CD3 (clone 17A2, Biolegend), CD4 (clone GK1.5, Biolegend), 
CD8a (clone 53-6.7, Biolegend), FOXP3 (clone MF-14, Biolegend), Mouse IFN- $\gamma$ (clone XMG1.2, Biolegend) and FOXP3 (clone MF-14 Biolegend).

In vitro monocyte trained immunity experiment: In vitro training of primary human monocytes was performed as described earlier ${ }^{47}$. Briefly, PBMCs were isolated from healthy donors (leukopaks). Following magnetic separation, CD14 ${ }^{+}$monocytes were seeded in $10 \mathrm{~mm}^{3}$ tissue culture dishes for 3 hours in warm RPMI 1640 media supplemented with $10 \%$ FBS at $37^{\circ} \mathrm{C}$ with $5 \% \mathrm{CO}_{2}$. Non-adherent cells were removed by washing cells using warm PBS. Monolayer culture of human monocytes was infected with BCG-WT and BCG-disA-OE strains at 5:1 (monocyte versus BCG) MOls for 4 hours in presence of RPMI 1640 supplemented with $10 \%$ FBS. Noninternalized bacilli were washed out using warm PBS and subsequently incubated for 24 hours. Cells were again washed using warm PBS and fresh warm RPMI 1640 media was added. For the following 5 days, cells were allowed to rest with a PBS wash and addition of fresh media every $2^{\text {nd }}$ day. Cells were re-stimulated on day 6 with RPMI 1640 supplemented with $10 \%$ FBS (negative control, without training) or TLR1/2 agonist, Pam3Cys (Cat. tlrl-pms, InvivoGen). Following stimulation, for $24 \mathrm{~h}$, culture supernatants were collected, filter sterilized and quickly snap-frozen $\left(-80^{\circ} \mathrm{C}\right)$ for cytokine measurement. Cells were harvested for chromatin immunoprecipitation (ChIP) experiments to measure epigenetic changes on gene promoters.

Chromatin immunoprecipitation (ChIP): Human monocytes were fixed with a final concentration of $1 \%$ formaldehyde for 10 minutes at room temperature. Cell fixation was stopped using $125 \mathrm{mM}$ glycine (Cat no. 50046, Sigma-Aldrich, USA), followed by sonication to fragment cellular DNA to an average size between 300 to 600 bp using Qsonica Sonicator Q125 (Cat. 15338283, Thermo Fisher Scientific). Sonicated cell lysates were subjected to immunoprecipitation (IP) by overnight incubation with recommended concentration of primary antibodies [(Histone H3K9me3 (H3K9 Trimethyl) Polyclonal Antibody cat. A-4036-100, 
epigentek); Anti-Histone H3 (tri methyl K4) antibody - ChIP Grade (ab8580), abcam)] in presence of magnetic Dynabeads (Cat no. 10004D, Thermo Fisher Scientific, USA) at $4^{\circ} \mathrm{C}$. Non-bound material was removed by sequentially washing the Dynabeads with lysis buffer, chromatin IP (ChIP) wash buffer and Tris-EDTA (TE buffer). DNA elution was done using ChIP elution buffer. Amplification of different segments of the regulatory regions of immunity genes was carried out using qPCR using specific primers. Reactions were normalized with input DNA while beads served as negative control. Details of all primary antibodies and sequence of primers have been given in supplementary table. S2.

Targeted Metabolite analysis with LC-MS/MS: Targeted metabolite analysis was performed with liquid-chromatography tandem mass spectrometry (LC-MS/MS) as described earlier ${ }^{48}$. Metabolites from cells or snap-frozen xenograft tumor tissue were extracted with $80 \%(\mathrm{v} / \mathrm{v})$ methanol solution equilibrated at $-80^{\circ} \mathrm{C}$, and the metabolite-containing supernatants were dried under nitrogen gas. Dried samples were re-suspended in $50 \%(\mathrm{v} / \mathrm{v})$ acetonitrile solution and $4 \mathrm{ml}$ of each sample were injected and analyzed on a 5500 QTRAP triple quadrupole mass spectrometer (AB Sciex) coupled to a Prominence ultra-fast liquid chromatography (UFLC) system (Shimadzu). The instrument was operated in selected reaction monitoring (SRM) with positive and negative ion-switching mode as described. This targeted metabolomics method allows for analysis of over two hundred of metabolites from a single 25-min LC-MS acquisition with a 3-ms dwell time and these analyzed metabolites cover all major metabolic pathways. The optimized MS parameters were: ESI voltage was $+5,000 \mathrm{~V}$ in positive ion mode and $-4,500 \mathrm{~V}$ in negative ion mode; dwell time was 3ms per SRM transition and the total cycle time was 1.57 seconds. Hydrophilic interaction chromatography (HILIC) separations were performed on a Shimadzu UFLC system using an amide column (Waters XBridge BEH Amide, 2.1 x 150 mm, $2.5 \mu \mathrm{m})$. The LC parameters were as follows: column temperature, $40{ }^{\circ} \mathrm{C}$; flow rate, $0.30 \mathrm{ml} / \mathrm{min}$. Solvent A, Water with $0.1 \%$ formic acid; Solvent B, Acetonitrile with $0.1 \%$ formic acid; A non-linear 
gradient from $99 \%$ B to $45 \%$ B in 25 minutes with 5 min of post-run time. Peak integration for each targeted metabolite in SRM transition was processed with MultiQuant software (v2.1, AB Sciex). The preprocessed data with integrated peak areas were exported from MultiQuant and reimported into Metaboanalyst software for further data analysis (e.g. statistical analysis, fold change, principle components analysis, etc.).

Histologic analyses and immunohistochemistry (IHC): For histologic analyses, a portion of bladder was formalin fixed and paraffin embedded. Sections of $5 \mu$ in thickness on glass slides were stained with hematoxylin-eosin for classification according to the World Health Organization/International Society of Urological Pathological consensus as described earlier ${ }^{27}$. Tumor staging was performed by 2 board certified genitourinary pathologists (A.S.B., A.M.). Specimens were classified based on the percentage of involvement of abnormal tissue $(1=10 \%$ involvement, 2 = $20 \%$ involvement, and so forth). For IHC staining, high-temperature antigen retrieval $\left(18-23\right.$ psi $\left./ 126{ }^{\circ} \mathrm{C}\right)$ was performed by immersing the slides in Trilogy (Cell Marque). Endogenous peroxidase activity was blocked for 5 min in using Dual Endogenous Enzyme Block (Cat. S2003, Dako). Primary Antibodies used included Ki67 (1:50, Cat. ab16667; Abcam), CD68 (1:250, Cat. MCA341R; Serotec), CD86 (1:100, Cat. bs-1035R; Bioss) and CD206 (1:10K, Cat. ab64693; Abcam). For Ki67, slides were stained with ImmPACT DAB (Vector Labs) for 3 min and counterstained with haematoxylin (Richard-Allen). Dual staining for CD68/CD206 and CD68/CD86 was achieved by first staining for CD68 with Impact DAB (Vector Labs) followed by secondary antigen retrieval and incubation as above with either CD86 or CD206 and visualized with ImmPACT AEC (Vector Labs). For each section, Ki67 expression was scored as a percentage of positive cells in the urothelium. Dual stains for CD68/CD86 and CD68/CD206 were scored based on positive clusters of cells for each marker $(0=$ no staining, $1=$ rare isolated cells positive, 2 = clusters of up to 10 positive cells, $3=$ clusters of $>10$ positive cells) . 


\section{In vivo experiments:}

Intravesical BCG treatment in carcinogen induced NMIBC rat model: The induction of urothelial cancer in rats and subsequent treatment of intravesical BCG were performed as previously described ${ }^{27}$. Briefly, N-methyl-N-nitrosourea (MNU) instillations were given every other week for a total of 4 instillations. Fischer 344 female rats age 7 weeks (Harlan, avg. weight 160g) were anesthetized with $3 \%$ isoflurane. After complete anesthesia, a $20 \mathrm{G}$ angiocatheter was placed into the rat's urethra. MNU (1.5mg/kg) (Spectrum) dissolved in $0.9 . \%$ sodium chloride was then instilled and the catheter removed, with continued sedation lasting for 60 minutes to prevent spontaneous micturition and allow absorption. Eighteen weeks after the first MNU instillation, intravesical treatment with PBS or $5 \times 10^{6} \mathrm{CFU}$ of each BCG strain (0.3ml via a $20 \mathrm{G}$ angiocatheter) was administered weekly for a total of 6 doses. Rodents were sacrificed $2 \mathrm{~d}$ after the last intravesical treatment, and bladders were harvested within 48 hours of the last BCG instillation for mRNA and protein expression analysis as well as histological evaluation.

BCG infection of BALB/c mice and CFU enumeration: To determine the lung bacillary burden of wild-type and BCG-disA-OE strains 6-week-old female BALB/c mice were exposed using the aerosol route in a Glasscol inhalation exposure system (Glasscol). The inoculum implanted in the lungs at day 1 ( $n=3$ mice per group) in female BALB/c mice was determined by plating the whole lung homogenate on $7 \mathrm{H} 11$ selective plates containing carbenicillin (50 mg/ml), Trimethoprim (20 $\mathrm{mg} / \mathrm{ml})$, Polymyxin B (25 mg/ml) and Cycloheximide $(10 \mathrm{mg} / \mathrm{ml})$. Following infection, mice lungs were harvested ( $n=5$ animals/group), homogenized in their entirety in sterile PBS and plated on $7 \mathrm{H} 11$ selective plates at different dilutions. The $7 \mathrm{H} 11$ selective plates were incubated at $37{ }^{\circ} \mathrm{C}$ and single colonies were enumerated at week 3 and 4 . Single colonies were expressed at log CFU per organ.

SCID Mice time to death study: The virulence testing of BCG-WT and BCG-disA-OE strains was done in severely compromised immunodeficient mice aerosol infection model as described 
previously. The inoculum implanted in the lungs at day 1 ( $n=3$ animals per group) was determined by plating the whole lung homogenate on $7 \mathrm{H} 11$ selective plates. For time to death analyses $(\mathrm{n}=$ 10 animals per group) infected animal were monitored until their death.

Syngeneic MB49 model of urothelial cancer: MB49 tumor cells are urothelial carcinoma line derived from an adult C57BL/6 mouse by exposure of primary bladder epithelial cell explant to 7,12-dimethylbenz[a]anthracene (DMBA) for 24 hours followed by a long-term culture ${ }^{79}$. Before implantation, MB49 cells were cultured as monolayers in RPMI 1640 media supplemented with $10 \%$ FBS and $1 \%$ streptomycin/penicillin at $37^{\circ} \mathrm{C}$ with $5 \% \mathrm{CO}_{2}$. Cells were harvested using Trypsinization and cell viability was determined using Trypan blue dye. Live MB49 cells were resuspended in sterile PBS and adjusted at $1 \times 10^{5}$ live cells per $100 \mu$ l. Female C57BL/6J mice, age 4-6 weeks (Charles River Laboratories) were subcutaneously injected with $1 \times 10^{5}$ MB49 cells in the right flank of hind leg. Tumor growth was monitored every $2^{\text {nd }}$ day to observe the increase the tumor burden at the time of treatment initiation. Once palpable tumor developed ( 7 to 9 days, average volume $\sim 30 \mathrm{~mm}^{3}$ ), $1 \times 10^{6}$ bacilli of BCG-WT or BCG-disA-OE in a total $50 \mu \mathrm{I}$ PBS was injected intratumorally (Fig. 1h). A total of 4 intratumoral injections of BCG was given every $3^{\text {rd }}$ day. Tumors were measured by electronic caliper, and tumor volume was calculated using the following equation: tumor volume $=$ length $x$ width $x$ height $x 0.5326$. Mice were killed at specified time, and tumors and spleens were collected after necropsy for single cell preparation.

\section{Figure legends (Main Figures):}

Figure 1. BCG-disA-OE elicits improved antitumor efficacy over BCG-WT in pre-clinical models of urothelial cancer. a. Schematic diagram of the MNU rat model of NMIBC. b. mRNA levels for proinflammatory cytokines (IFN- $\beta$, IFN- $\gamma$, TNF- $\alpha$ and IL-1 $\beta$ ), regulatory chemokines (CXCL10, MCP-1 and MIP-1 $\alpha$ ), immunosuppressive M2 macrophage cytokines (IL- 
10 and TGF- $\beta$ ) and M1 tumoricidal effectors (iNOS) in whole bladders at necropsy (wk 23) measured by qRT-PCR relative to GAPDH (5 animals/group). c. Representative H \& E staining showing highest pathology grade for each group [control, untreated MNU bladder, BCG-WT (Past and Tice), and BCG-disA-OE (Past and Tice)-12-16 animals/group]. d. Tumor involvement values at necropsy (7-11 animals/group). e. Highest tumor stage at necropsy. f. Representative immunohistochemistry and bar graph of rat bladder tissue at necropsy stained for Ki67. g. Representative immunohistochemical co-staining and line graph for CD68 (brown), CD86 (M1 macrophages; red) and CD206 (M2 macrophages; red) in rat bladder tissues at necropsy; $\mathbf{h}$. Schematic diagram of the MB49 syngeneic mouse model of urothelial cancer. i. MB49 tumor volume and tumor weight at time of necropsy (9 animals/group). j. Tumor infiltrating M1 and M2 macrophages and CD4 and CD8 T cell lymphocyte types at necropsy in the MB49 model. * $p<$ $0.05,{ }^{* *} p<0.01,{ }^{* * *} p<0.001,{ }^{* * *} p<0.0001$ by 2 -tailed Student's t-test. \#\# $p<0.01$ by 1 -way ANOVA.

Figure 2. BCG-disA-OE elicits greater pro-inflammatory cytokine responses than BCG-WT in primary human and murine macrophages and dendritic cells in vitro. a. Schematic diagram. b. IRF3 induction measured in RAW-Lucia ISG reporter macrophages. c. IFN- $\beta$ levels in murine BMDM from wild type and STING ${ }^{-/-}$mice. d. IFN- $\beta$, TNF- $\alpha$, and IL-6 levels from primary human and murine macrophages and dendritic cells and the J774.1 murine macrophage cell line. e. mRNA levels for Th1 cytokines and chemokines in murine BMDM relative to $\beta$-actin. Cytokine levels were measured by ELISA after $24 \mathrm{hr}$ exposures at a MOI of $20: 1$. Data are SEM $(n=3$ replicates). ${ }^{*} p<0.05,{ }^{* *} p<0.01,{ }^{* * *} p<0.001,{ }^{* * * *} p<0.0001$ by 2 -tailed Student's t-test. Data shown are for BCG-Tice; similar findings were observed for BCG-Pasteur as shown in Fig. S4.

Figure 3. BCG-disA-OE elicits greater pro-inflammatory cytokine responses and autophagy than BCG-WT in human and murine urothelial carcinoma cells. 
a-d. TNF- $\alpha$, IL-6, IL-1 $\beta$ and IFN- $\gamma$ levels in urothelial carcinoma cell lines: 5637 (human epithelial high grade carcinoma), RT4 (human transitional low grade carcinoma), NBT II (carcinogeninduced rat tumor), MB49, UPPL1595, and BBN975 (all carcinogen-induced mouse tumors) Cytokine levels were measured by ELISA after $24 \mathrm{hr}$ exposures at MOI of 20:1. Data are SEM ( $\mathrm{n}$ $=3$ replicates). $\quad$ e. Autophagy induction in the 5637 human urothelial carcinoma cells in representative confocal photomicrographs. Colocalization of FITC-labeled BCG strains (green), LC3B autophagic puncta (red) appears in yellow; nuclei are blue. Cells were fixed using 4\% paraformaldehyde 3h after infection (MOI 10:1), and images obtained with an LSM700 confocal microscope and Fiji software processing. ${ }^{*} p<0.05$, ${ }^{* *} p<0.01,{ }^{* * *} p<0.001,{ }^{* * * *} p<0.0001$ by 2-tailed Student's t-test. Data shown are for BCG-Tice; similar findings were observed for BCGPasteur as shown in Fig. S7.

Figure 4. BCG-disA-OE elicits greater macrophage re-programming, phagocytic activity, and autophagy than BCG-WT in human and murine macrophages. a. Percentages of M1and M2-macrophages and M-MDSCs arising from primary murine macrophages. b. Percentages of inflammatory, TNF- $\alpha^{+} \mathrm{M} 1$, and IL- $6^{+} \mathrm{M} 1$ macrophages, and c. M2 and IL-10 ${ }^{+} \mathrm{M} 2$ macrophages arising from primary human macrophages. Data were collected after $24 \mathrm{hr}$ exposures at MOI of 20:1 as determined by flow cytometry using gating schemes shown in Fig. S8-S13. Data are SEM ( $n=3$ replicates). d. Phagocytic activity in human primary macrophages in representative confocal photomicrographs showing intracellular uptake of FITC-labeled IgG-opsonized latex beads (green) with nuclei stained blue. e. Autophagy induction and f. quantification by BCGLC3B colocalization in primary murine macrophages shown by representative confocal photomicrographs. Autophagy was measured by LC3B puncta or g. p62 colocalization with BCG appearing in yellow. FITC-labeled BCG strains are stained green, LC3B or p62 autophagic puncta (red), and nuclei blue. h. Quantification of BCG-p62 colocalization. Cells were fixed using 4\% paraformaldehyde $6 \mathrm{~h}$ after infection (MOI 10:1), and images obtained with an LSM700 
confocal microscope and Fiji software processing. Quantification was by mean fluorescence intensity. ${ }^{*} p<0.05,{ }^{* *} p<0.01,{ }^{* * *} p<0.001,{ }^{* * *} p<0.0001$ by 2 -tailed Student's t-test. Data shown are for BCG-Tice; similar findings were observed for BCG-Pasteur as shown in Fig. \$9 and S11.

Figure 5. Compared with BCG-WT, BCG-disA-OE is a more potent inducer of epigenetic and metabolomic changes characteristic of trained immunity in primary human monocytes. a. mRNA levels of TNF-a and IL-6 in primary human monocytes (6 healthy donors) relative to RNU6A after $24 \mathrm{hr}$ exposures at a MOI of 10:1. b. Schematic diagram of ex vivo monocyte training. c. Relative levels of the H3K4me3 chromatin activation mark or (d) the H3K9me3 chromatin repression mark retrieved from the TNF- $\alpha$ and IL-6 promoter regions of primary human monocytes from 4 healthy donors (D1-D4) determined by ChIP-PCR assay on day 7. e-f. Secreted cytokines (TNF- $\alpha$ and IL-6) following BCG training and re-stimulation. Monocytes were initially challenged on day 0 with a $24 \mathrm{hr}$ exposure to the BCG strains at a MOI of $10: 1$ followed by washing. After five days of rest they were treated for $24 \mathrm{~h}$ with either a sham second stimulus (RPMI) or the TLR1/2 agonist Pam3CSK4. g-i. Metabolite levels determined by LCMS in human or murine MDM determined $24 \mathrm{hr}$ after exposure to BCG strains or heat-killed controls. Cartoon diagram (i) showing key metabolites upregulated (red arrow upward) in BCGdisA-OE infected macrophages relative to BCG-WT infected macrophages. $\quad{ }^{* *} p<0.01$ by $2-$ tailed Student's t-test.

Figure 6. BCG-disA-OE is less pathogenic than BCG-WT in two mouse models and elicits a greater proinflammatory cytokine levels in vivo. a. Schematic diagram of the immunocompetent BALB/c mouse challenge model. b. Lung colony forming unit (CFU) counts at day 1 and day 28 (5 animals/group). Data are S.E.M. c. Levels of the pro-inflammatory cytokines IFN-b, TNF-a, IL-6, and IFN-g in mouse lungs and (d) spleens determined by ELISA at 
day 28 (4 animals/group). Data are S.E.M. e. Schematic diagram of the immunocompromised SCID mouse challenge model. f. Percent survival of SCID mice following low dose challenge 10 animals/group). The day 1 lung CFU counts are shown at right. ${ }^{*} p<0.05,{ }^{* *} p<0.01,{ }^{* * *} p$ $<0.001,{ }^{* * *} p<0.0001$ by 2-tailed Student's t-test.

\section{Supplementary Figure legends:}

\section{Supplementary Figure 1. Confirmation of M.tb-disA overexpression BCG-disA-OE and} induction of IRF signaling. a. mRNA level of disA in log-phase BCG cultures relative to $M$. tuberculosis sigA (Rv2703). b. IRF3 induction measured in RAW-Lucia ISG reporter macrophages. IRF quantification was carried out in culture supernatant of macrophages infected at a MOI of $20: 1$ for 24 hrs. ${ }^{* * *} p<0.001,{ }^{* * *} p<0.0001$ by 2 -tailed Student's t-test.

Supplementary Figure 2. BCG-disA-OE causes reduced tumor outgrowth in syngeneic MB49 model of urothelial cancer. a. M1 macrophage cytokines in MNU rat bladder, spleen and blood serum (5 animals / group). b. Tumors after necropsy (9 animals / group). c. Representative H \& E staining showing necrotic area and congestion in MB49 tumors. d. Body and spleen weight after necropsy ( 9 animals / group). ${ }^{*} p<0.05,{ }^{* *} p<0.01$ by 2 -tailed Student's t-test.

Supplementary Figure 3. BCG-disA-OE causes stronger recruitment of effector T cells and strongly dampens immunosuppressive Treg lymphocytes. a. Increased percentage of CD4 ${ }^{+}$ and $\mathrm{CD}^{+} \mathrm{T}$ cells in MB49 tumors and mouse spleens (9 animals / group). b. Decreased percentage of CD4+ ${ }^{+}$Treg in tumor and spleen of MB49 tumor bearing animals ( 9 animals / group). ${ }^{*} p<0.05,{ }^{* *} p<0.01,{ }^{* * *} p<0.001,{ }^{* * *} p<0.0001$ by 2-tailed Student's t-test.

Supplementary Figure 4. BCG-disA-OE elicits stronger IFN- $\beta$, pro-inflammatory cytokines and chemokines than BCG-WT in primary human and murine macrophages and dendritic cells. a. Schematic diagram b. IFN- $\beta$ levels in resting and IFN- $\gamma$ primed BMDMs. c. IFN- $\beta$, TNF$\alpha$, and IL-6 levels in human and murine primary macrophages, murine primary dendritic cells and 
J774.1 murine macrophage cell line. d. mRNA levels of il6, il12 and mcp1 in IFN- $\gamma$-activated primary human macrophages relative to $\beta$-actin. e. MCP-1 levels in murine primary macrophages. Cytokines and chemokine levels were measured by ELISA after 24 hr exposures at a MOI of 20:1. SEM ( $n=3$ replicates). Gene expression analyses for cytokines and chemokines was done after $6 \mathrm{hr}$ exposure. ${ }^{*} \mathrm{p}<0.05,{ }^{* *} \mathrm{p}<0.01,{ }^{* * *} \mathrm{p}<0.001,{ }^{* * * *} \mathrm{p}<0.0001$ by 2 -tailed Student's t-test.

Supplementary Figure 5. BCG-disA-OE is less toxic to macrophages than that of WT-BCG.

a-b. Percentage late apoptotic murine primary macrophages and J774.1 macrophage cell line. Data was collected after $24 \mathrm{hr}$ exposure at a MOI of 20:1 as determined by flow cytometry. SEM ( $n=3$ replicates). ${ }^{*} p<0.05$ by 2 -tailed Student's t-test.

Supplementary Figure 6. BCG internalization and viability of urothelial carcinoma cells. a. Internalization of WT and BCG-disA-OE (Tice) in human urothelial carcinoma cell, 5637. b. Viability of human urothelial carcinoma cells measured using CellTiter-Glo Luminescent Cell Viability assay after BCG exposure at different MOls. For confocal microscopy, FITC-labeled BCG strains are stained green and nuclei are stained blue (Hoechst). Cells were fixed using $4 \%$ paraformaldehyde30 mins after infection (MOI 5:1), and images (63X) were obtained using an LSM700 confocal microscope and Fiji software processing.

\section{Supplementary Figure 7. BCG-disA-OE elicits greater pro-inflammatory cytokine responses than BCG-WT in human and rodent urothelial carcinoma cells.}

a-b. TNF- $\alpha$, IL-1 $\beta$, IFN- $\gamma$ and IL-6 levels in urothelial carcinoma cell lines: 5637 (human epithelial high-grade carcinoma), BBN975, MB49 and UPPL1595 (all carcinogen induced mouse tumor cells), NBT2 (carcinogen-induced rat tumors). Cytokine levels were measured by ELISA after 24 hr exposures at MOI of 20:1. Data are SEM ( $n=3$ replicates). ${ }^{*} p<0.05,{ }^{* *} p<0.01,{ }^{* * *} p<$ $0.001,{ }^{* * *} p<0.0001$ by 2 -tailed Student's t-test. 
Supplementary Figure 8. Representative gating scheme for identification different myeloid populations in mouse BMDMs. a. Schematic of generation of BMDMs. b. Representative gating scheme for identification of different myeloid cells. Briefly, leukocyte lineage was selected by gating SSC-A against $\mathrm{CD} 45^{+}$populations on live cells. $\mathrm{CD} 11 \mathrm{~b}^{+} \mathrm{F} 4 / 80^{+}$macrophages were identified out of $C D 45^{+}$population. $\mathrm{CD} 11 \mathrm{~b}^{+} \mathrm{F} 4 / 80^{+}$macrophages were divided into MHC class II (I-a/l-e) and CD124+CD206+ populations. Expression of TNF- $\alpha$ (M1 macrophages) and IL-10 (M2 macrophages) were determined on MHC class II subsets and CD124 ${ }^{+} \mathrm{CD} 206^{+}$subsets respectively (Related to Fig. $4 a)$.

Supplementary Figure 9. BCG-disA-OE induces macrophage reprogramming and favors stronger M1 macrophage shift in murine BMDMs. a-b. Percentage TNF- $\alpha^{+}$M1 macrophages $\left(\mathrm{MHC}\right.$ Class $\left.\mathrm{II}^{+} \mathrm{CD} 11 \mathrm{~b}^{+} \mathrm{F} 4 / 80^{+}\right)$and corresponding representative FACS plots. c-d. Percentage M2 macrophages $\left(C D 206^{+} C D 124^{+}\right)$and corresponding representative FACS plots. e-f. Percentage IL-10+ $\mathrm{M} 2$ macrophages $\left(\mathrm{CD} 206^{+} \mathrm{CD} 124^{+}\right)$and corresponding representative FACS plots. Data are SEM $\left(n=3\right.$ replicates). ${ }^{*} p<0.05,{ }^{* *} p<0.01,{ }^{* * *} p<0.001,{ }^{* * *} p<0.0001$ by $2-$ tailed Student's t-test. (Related to Fig. 4a).

Supplementary Figure 10. Gating scheme showing identification of myeloid-derived suppressor cell populations in primary mouse macrophages after BCG exposure. Leukocyte lineage was determined on live cells by gating SSC-A against CD45+ myeloid cells. Myeloid cells were differentiated into $\mathrm{CD} 11 \mathrm{~b}^{+} \mathrm{F} 4 / 80^{+}$macrophages out of which $\mathrm{CD} 11 \mathrm{~b}^{+} \mathrm{F} 4 / 80^{-}$ myeloid population was divided into Ly6C and Ly6G. Next, the Ly6C ${ }^{(\text {hi) }}$ Ly6G immunosuppressive myeloid-derived suppressor cell populations were looked for IL-10 positivity (Related to Fig. 4a). Supplementary Figure 11. Immunosuppressive monocytic-MDSCs (M-MDSCs) populations murine primary macrophages after BCG exposure. a-b. Percentage of M-MDSCs and corresponding representative FACS plot. c-d. Percentage of $\mathrm{IL}-10^{+}$immunosuppressive $\mathrm{M}$ - 
MDSCs and corresponding representative FACS plots. Data are SEM ( $n=3$ replicates). ${ }^{* *} p<$ 0.01 by 2-tailed Student's t-test. (Related to Fig. 4a).

\section{Supplementary Figure 12. BCG-disA-OE strongly induces inflammatory human}

macrophages. a. Gating strategy for M1 macrophages. b. Gating strategy for M2 macrophages.

c. Representative FACS plot showing percentage of classically activated (CD14 ${ }^{+}$CD16 $)$ macrophages after BCG exposure. (Related to Figure 4b).

Supplementary Figure 13. BCG-disA-OE causes strong M1 macrophage shift in human primary macrophages. a. FACS plot showing inflammatory M1 macrophages (CD14 ${ }^{+}$CD16 $\left.{ }^{-}\right)$. b. FACS plots showing immunosuppressive $\mathrm{M} 2$ macrophages $\left(\mathrm{CD} 206^{+} \mathrm{CD} 163^{+}\right.$of $\left.\mathrm{CD} 14^{+} \mathrm{CD} 16^{+}\right)$after $\mathrm{BCG}$ exposure. (Related to Figure $\mathbf{4 b}$ ).

Supplementary Figure 14. Sting agonist c-di-AMP causes induction of macrophage activation. Human macrophages were transfected with c-di-AMP for $24 \mathrm{~h}$ and phagocytosis of FITC-labeled IgG opsonized latex beads (green) was visualized using confocal microscopy on live cells. Hoechst was used for nuclear staining (blue). Images were acquired using LSM700 confocal microscope at $63 \mathrm{X}$ magnification. Images were process using Fiji software.

Supplementary Figure 15. BCG-disA-OE (Pasteur) is less pathogenic that BCG-WT in mouse models and elicits invokes stronger proinflammatory cytokines in vivo. a. Lung colony forming unit (CFU) counts at day 1 and day 28 (5 animals/group). Data are S.E.M. b. Levels of the pro-inflammatory cytokines IFN- $\beta$, TNF- $\alpha$, IL-6, and IFN- $\gamma$ in mouse lungs and spleens determined by ELISA at day 28 (4 animals/group). Data are S.E.M. ( $\mathrm{n}=5$ animals for CFU; $n=3$ animals for day 1 lung implantation). c. Percent survival of SCID mice following low dose challenge (10 animals/group). The day 1 lung CFU counts are shown at right. ${ }^{*} p<0.05$, ${ }^{* *} p<0.01,{ }^{* * *} p<0.001,{ }^{* * * *} p<0.0001$ by 2 -tailed Student's t-test. 


\section{ACKNOWLEDGEMENTS:}

The authors gratefully acknowledge the financial support of NIH AI 37856, Tedco awards MII4181, MII-5072, and awards from the Willowcroft Foundation and the Cigarette Restitution Fund of Maryland. The authors also thank Dr. Gregory Joice and Emily Juzwiak for experimental assistance and Dr. Geetha Srikrishna for editorial assistance.

\section{AUTHORS CONTRIBUTIONS:}

AKS, WRB and TJB co-led the study through conceptualization, design, oversight, and the interpretation of results. WRB and TJB obtained funding for the study. AKS and MP designed, conducted, and interpreted the results of experiments. KAL, TY, AM, ASB, LZ, and PP conducted and interpreted the results of experiments. JDP, MLK, and DM assisted in the design of experiments and provided key expert advice. AKS, WRB, and TJB wrote the manuscript. AKS, MP, KAL, TY, AM, ASB, LZ, PP, JDP, MLK, DM, DMP, WRB, and TJB revised and edited the manuscript. AKS, WRB, and TJB designed and produced figures for this manuscript.

\section{COMPETING FINANCIAL INTERESTS:}

WRB and TJB are co-founders of OncoSTING, LLC which holds rights to commercialize BCG$\operatorname{dis} A-\mathrm{OE}$.

\section{REFERENCES:}

1. Becker, R. E. N., Kates, M. R. \& Bivalacqua, T. J. Identification of Candidates for Salvage Therapy: The Past, Present, and Future of Defining Bacillus Calmette-Guérin Failure. Urol. Clin. North Am. 47, 15-21 (2020). 
2. Pettenati, C. \& Ingersoll, M. A. Mechanisms of BCG immunotherapy and its outlook for bladder cancer. Nat Rev Urol 15, 615-625 (2018).

3. Ingersoll, M. A. \& Albert, M. L. From infection to immunotherapy: host immune responses to bacteria at the bladder mucosa. Mucosal Immunol 6, 1041-1053 (2013).

4. Suttmann, H. et al. Neutrophil granulocytes are required for effective Bacillus Calmette-Guérin immunotherapy of bladder cancer and orchestrate local immune responses. Cancer Res. 66, 8250-8257 (2006).

5. Brandau, S. et al. NK cells are essential for effective BCG immunotherapy. Int. J. Cancer 92, 697-702 (2001).

6. Ratliff, T. L., Ritchey, J. K., Yuan, J. J., Andriole, G. L. \& Catalona, W. J. T-cell subsets required for intravesical BCG immunotherapy for bladder cancer. J. Urol. 150, 1018-1023 (1993).

7. Pichler, R. et al. Tumor-infiltrating immune cell subpopulations influence the oncologic outcome after intravesical Bacillus Calmette-Guérin therapy in bladder cancer. Oncotarget 7, $39916-39930$ (2016).

8. Butkeviciute, E., Jones, C. E. \& Smith, S. G. Heterologous effects of infant BCG vaccination: potential mechanisms of immunity. Future Microbiol 13, 1193-1208 (2018).

9. Netea, M. G. \& van Crevel, R. BCG-induced protection: effects on innate immune memory. Semin. Immunol. 26, 512-517 (2014).

10. Arts, R. J. W. et al. BCG Vaccination Protects against Experimental Viral Infection in Humans through the Induction of Cytokines Associated with Trained Immunity. Cell Host Microbe 23, 89-100.e5 (2018).

11. Kaufmann, E. et al. BCG Educates Hematopoietic Stem Cells to Generate Protective Innate Immunity against Tuberculosis. Cell 172, 176-190.e19 (2018).

12. Arts, R. J. W. et al. Immunometabolic Pathways in BCG-Induced Trained Immunity. Cell Rep $17,2562-2571(2016)$. 
13. Kleinnijenhuis, J. et al. Bacille Calmette-Guerin induces NOD2-dependent nonspecific protection from reinfection via epigenetic reprogramming of monocytes. Proc. Natl. Acad. Sci. U.S.A. 109, 17537-17542 (2012).

14. Kleinnijenhuis, J. et al. BCG-induced trained immunity in NK cells: Role for non-specific protection to infection. Clin. Immunol. 155, 213-219 (2014).

15. Netea, M. G. et al. Defining trained immunity and its role in health and disease. Nat. Rev. Immunol. (2020) doi:10.1038/s41577-020-0285-6.

16. Sun, L., Wu, J., Du, F., Chen, X. \& Chen, Z. J. Cyclic GMP-AMP synthase is a cytosolic DNA sensor that activates the type I interferon pathway. Science 339, 786-791 (2013).

17. Hansen, K. et al. Listeria monocytogenes induces IFN $\beta$ expression through an IFI16-, cGASand STING-dependent pathway. EMBO J. 33, 1654-1666 (2014).

18. Dey, B. et al. A bacterial cyclic dinucleotide activates the cytosolic surveillance pathway and mediates innate resistance to tuberculosis. Nat. Med. 21, 401-406 (2015).

19. Barker, J. R. et al. STING-dependent recognition of cyclic di-AMP mediates type I interferon responses during Chlamydia trachomatis infection. mBio 4, e00018-00013 (2013).

20. Gutjahr, A. et al. The STING ligand cGAMP potentiates the efficacy of vaccine-induced CD8+ T cells. JCI Insight 4, (2019).

21. Barber, G. N. STING: infection, inflammation and cancer. Nat. Rev. Immunol. 15, 760-770 (2015).

22. Corrales, L. et al. Direct Activation of STING in the Tumor Microenvironment Leads to Potent and Systemic Tumor Regression and Immunity. Cell Rep 11, 1018-1030 (2015).

23. Vatner, R. E. \& Janssen, E. M. STING, DCs and the link between innate and adaptive tumor immunity. Mol. Immunol. 110, 13-23 (2019).

24. Marloye, M., Lawler, S. E. \& Berger, G. Current patent and clinical status of stimulator of interferon genes (STING) agonists for cancer immunotherapy. Pharm Pat Anal 8, 87-90 (2019). 
25. Iurescia, S., Fioretti, D. \& Rinaldi, M. Targeting Cytosolic Nucleic Acid-Sensing Pathways for Cancer Immunotherapies. Front Immunol 9, 711 (2018).

26. Dey, R. J., Dey, B., Singh, A. K., Praharaj, M. \& Bishai, W. Bacillus Calmette-Guérin Overexpressing an Endogenous Stimulator of Interferon Genes Agonist Provides Enhanced Protection Against Pulmonary Tuberculosis. J. Infect. Dis. 221, 1048-1056 (2020).

27. Kates, M. et al. Intravesical BCG Induces CD4+ T-Cell Expansion in an Immune Competent Model of Bladder Cancer. Cancer Immunol Res 5, 594-603 (2017).

28. Yoshida, T. et al. Ex vivo culture of tumor cells from N-methyl-N-nitrosourea-induced bladder cancer in rats: Development of organoids and an immortalized cell line. Urol. Oncol. 36, 160.e23-160.e32 (2018).

29. Kates, M. et al. Preclinical Evaluation of Intravesical Cisplatin Nanoparticles for Non-MuscleInvasive Bladder Cancer. Clin. Cancer Res. 23, 6592-6601 (2017).

30. Fulton, S. A. et al. Inhibition of major histocompatibility complex II expression and antigen processing in murine alveolar macrophages by Mycobacterium bovis BCG and the 19kilodalton mycobacterial lipoprotein. Infect. Immun. 72, 2101-2110 (2004).

31. Jing, W. et al. STING agonist inflames the pancreatic cancer immune microenvironment and reduces tumor burden in mouse models. J Immunother Cancer 7, 115 (2019).

32. Francica, B. J. et al. TNFa and Radioresistant Stromal Cells Are Essential for Therapeutic Efficacy of Cyclic Dinucleotide STING Agonists in Nonimmunogenic Tumors. Cancer Immunol Res 6, 422-433 (2018).

33. Bevers, R. F. M., Kurth, K.-H. \& Schamhart, D. H. J. Role of urothelial cells in BCG immunotherapy for superficial bladder cancer. Br. J. Cancer 91, 607-612 (2004).

34. Jackson, A. M. et al. Changes in urinary cytokines and soluble intercellular adhesion molecule-1 (ICAM-1) in bladder cancer patients after bacillus Calmette-Guérin (BCG) immunotherapy. Clin. Exp. Immunol. 99, 369-375 (1995). 
35. De Boer, E. C. et al. Induction of urinary interleukin-1 (IL-1), IL-2, IL-6, and tumour necrosis factor during intravesical immunotherapy with bacillus Calmette-Guérin in superficial bladder cancer. Cancer Immunol. Immunother. 34, 306-312 (1992).

36. Liu, D. et al. STING directly activates autophagy to tune the innate immune response. Cell Death Differ. 26, 1735-1749 (2019).

37. Watson, R. O. et al. The Cytosolic Sensor cGAS Detects Mycobacterium tuberculosis DNA to Induce Type I Interferons and Activate Autophagy. Cell Host Microbe 17, 811-819 (2015).

38. Suriano, F. et al. Tumor associated macrophages polarization dictates the efficacy of BCG instillation in non-muscle invasive urothelial bladder cancer. J. Exp. Clin. Cancer Res. 32, 87 (2013).

39. Gabrilovich, D. I. Myeloid-Derived Suppressor Cells. Cancer Immunol Res 5, 3-8 (2017).

40. Kumar, V., Patel, S., Tcyganov, E. \& Gabrilovich, D. I. The Nature of Myeloid-Derived Suppressor Cells in the Tumor Microenvironment. Trends Immunol. 37, 208-220 (2016).

41. Dahal, L. N. et al. STING Activation Reverses Lymphoma-Mediated Resistance to Antibody Immunotherapy. Cancer Res. 77, 3619-3631 (2017).

42. Corrales, L., McWhirter, S. M., Dubensky, T. W. \& Gajewski, T. F. The host STING pathway at the interface of cancer and immunity. J. Clin. Invest. 126, 2404-2411 (2016).

43. Zhu, Y. et al. STING: a master regulator in the cancer-immunity cycle. Mol. Cancer 18, 152 (2019).

44. Ohkuri, T. et al. Intratumoral administration of cGAMP transiently accumulates potent macrophages for anti-tumor immunity at a mouse tumor site. Cancer Immunol. Immunother. 66, 705-716 (2017).

45. Jagannath, C. et al. Autophagy enhances the efficacy of BCG vaccine by increasing peptide presentation in mouse dendritic cells. Nat. Med. 15, 267-276 (2009).

46. Crotzer, V. L. \& Blum, J. S. Autophagy and its role in MHC-mediated antigen presentation. J. Immunol. 182, 3335-3341 (2009). 
47. Bekkering, S. et al. In Vitro Experimental Model of Trained Innate Immunity in Human Primary Monocytes. Clin. Vaccine Immunol. 23, 926-933 (2016).

48. Leone, R. D. et al. Glutamine blockade induces divergent metabolic programs to overcome tumor immune evasion. Science 366, 1013-1021 (2019).

49. Andrejeva, G. \& Rathmell, J. C. Similarities and Distinctions of Cancer and Immune Metabolism in Inflammation and Tumors. Cell Metab. 26, 49-70 (2017).

50. Vitale, I., Manic, G., Coussens, L. M., Kroemer, G. \& Galluzzi, L. Macrophages and Metabolism in the Tumor Microenvironment. Cell Metab. 30, 36-50 (2019).

51. Antoni, S. et al. Bladder Cancer Incidence and Mortality: A Global Overview and Recent Trends. Eur. Urol. 71, 96-108 (2017).

52. Kamat, A. M. et al. Definitions, End Points, and Clinical Trial Designs for Non-Muscle-Invasive Bladder Cancer: Recommendations From the International Bladder Cancer Group. J. Clin. Oncol. 34, 1935-1944 (2016).

53. Lerner, S. P. et al. Clarification of Bladder Cancer Disease States Following Treatment of Patients with Intravesical BCG. Bladder Cancer 1, 29-30 (2015).

54. Kaye, D. R. et al. Costs of Cancer Care Across the Disease Continuum. Oncologist 23, 798805 (2018).

55. Correa, A. F. et al. The Role of Interferon in the Management of BCG Refractory Nonmuscle Invasive Bladder Cancer. Adv Urol 2015, 656918 (2015).

56. Lamm, D., Brausi, M., O’Donnell, M. A. \& Witjes, J. A. Interferon alfa in the treatment paradigm for non-muscle-invasive bladder cancer. Urol. Oncol. 32, 35.e21-30 (2014).

57. Plote, D. et al. Inhibition of urothelial carcinoma through targeted type I interferon-mediated immune activation. Oncoimmunology 8, e1577125 (2019).

58. Fahmi, T., Port, G. C. \& Cho, K. H. c-di-AMP: An Essential Molecule in the Signaling Pathways that Regulate the Viability and Virulence of Gram-Positive Bacteria. Genes (Basel) 8, (2017). 
59. Opoku-Temeng, C., Zhou, J., Zheng, Y., Su, J. \& Sintim, H. O. Cyclic dinucleotide (c-di-GMP, c-di-AMP, and cGAMP) signalings have come of age to be inhibited by small molecules. Chem. Commun. (Camb.) 52, 9327-9342 (2016).

60. Covián, C. et al. BCG-Induced Cross-Protection and Development of Trained Immunity: Implication for Vaccine Design. Front Immunol 10, 2806 (2019).

61. Saeed, S. et al. Epigenetic programming of monocyte-to-macrophage differentiation and trained innate immunity. Science 345, 1251086 (2014).

62. Penkov, S., Mitroulis, I., Hajishengallis, G. \& Chavakis, T. Immunometabolic Crosstalk: An Ancestral Principle of Trained Immunity? Trends Immunol. 40, 1-11 (2019).

63. Arts, R. J. W., Joosten, L. A. B. \& Netea, M. G. Immunometabolic circuits in trained immunity. Semin. Immunol. 28, 425-430 (2016).

64. van der Heijden, C. D. C. C. et al. Epigenetics and Trained Immunity. Antioxid. Redox Signal. 29, 1023-1040 (2018).

65. Mourits, V. P., Wijkmans, J. C., Joosten, L. A. \& Netea, M. G. Trained immunity as a novel therapeutic strategy. Curr Opin Pharmacol 41, 52-58 (2018).

66. Usher, N. T. et al. Association of BCG Vaccination in Childhood With Subsequent Cancer Diagnoses: A 60-Year Follow-up of a Clinical Trial. JAMA Netw Open 2, e1912014 (2019).

67. Netea, M. G., Joosten, L. A. B. \& van der Meer, J. W. M. Hypothesis: stimulation of trained immunity as adjunctive immunotherapy in cancer. J. Leukoc. Biol. 102, 1323-1332 (2017).

68. Stevens, W. B. C., Netea, M. G., Kater, A. P. \& van der Velden, W. J. F. M. 'Trained immunity': consequences for lymphoid malignancies. Haematologica 101, 1460-1468 (2016).

69. Hamada, A., Torre, C., Drancourt, M. \& Ghigo, E. Trained Immunity Carried by Non-immune Cells. Front Microbiol 9, 3225 (2018).

70. Pathria, P., Louis, T. L. \& Varner, J. A. Targeting Tumor-Associated Macrophages in Cancer. Trends Immunol. 40, 310-327 (2019). 
71. Buffen, K. et al. Autophagy controls BCG-induced trained immunity and the response to intravesical BCG therapy for bladder cancer. PLoS Pathog. 10, e1004485 (2014).

72. Yang, G. et al. Accumulation of myeloid-derived suppressor cells (MDSCs) induced by low levels of IL-6 correlates with poor prognosis in bladder cancer. Oncotarget 8, 38378-38388 (2017).

73. Schneider, A. K., Chevalier, M. F. \& Derré, L. The multifaceted immune regulation of bladder cancer. Nat Rev Urol 16, 613-630 (2019).

74. Thøstesen, L. M. et al. Neonatal BCG vaccination and atopic dermatitis before 13 months of age: A randomized clinical trial. Allergy 73, 498-504 (2018).

75. de Andrade, C. R. et al. Does BCG revaccination protect against the development of asthma? Respir Med 107, 317-319 (2013).

76. Zhang, C.-X. et al. STING signaling remodels the tumor microenvironment by antagonizing myeloid-derived suppressor cell expansion. Cell Death Differ. 26, 2314-2328 (2019).

77. Zarif, J. C. et al. A phased strategy to differentiate human CD14+monocytes into classically and alternatively activated macrophages and dendritic cells. BioTechniques 61, 33-41 (2016).

78. Rahman, A., Sobia, P., Gupta, N., Kaer, L. V. \& Das, G. Mycobacterium tuberculosis subverts the TLR-2-MyD88 pathway to facilitate its translocation into the cytosol. PLoS ONE 9, e86886 (2014).

79. Summerhayes, I. C. \& Franks, L. M. Effects of donor age on neoplastic transformation of adult mouse bladder epithelium in vitro. J. Natl. Cancer Inst. 62, 1017-1023 (1979). 
bioRxiv preprint doi: https://doi.org/10.1101/2020.04.25.061531; this version posted May 13, 2020. The copyright holder for this preprint (which

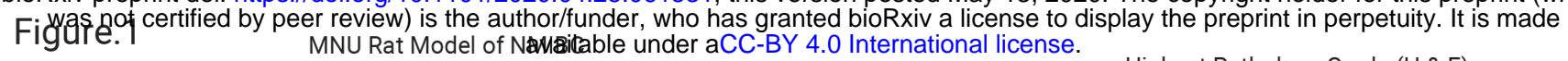

a

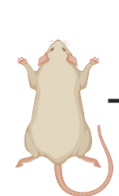

Fisher 344

Female Rat

(7 weeks)
MNU instillation Intravesical BCG instillation

$(4 \times 1.5 \mathrm{mg} / \mathrm{kg}) \quad\left(1 \times 10^{6}\right.$ bacilli $/ 50$ ul PBS $)$

\begin{tabular}{|c|c|}
\hline $\begin{array}{lccc} & 4 & 4 & 4 \\
\text { wo } & \text { w2 } & \text { w4 } & \text { w6 }\end{array}$ & 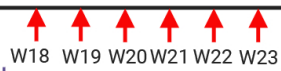 \\
\hline $\begin{array}{c}\text { Carcinogenesis } \\
\text { Phase }\end{array}$ & $\begin{array}{l}\text { Intravesical BCG } \\
\text { immunotherapy }\end{array}$ \\
\hline
\end{tabular}

MNU Rat Bladder (Post-BCG immunotherapy) (qPCR)

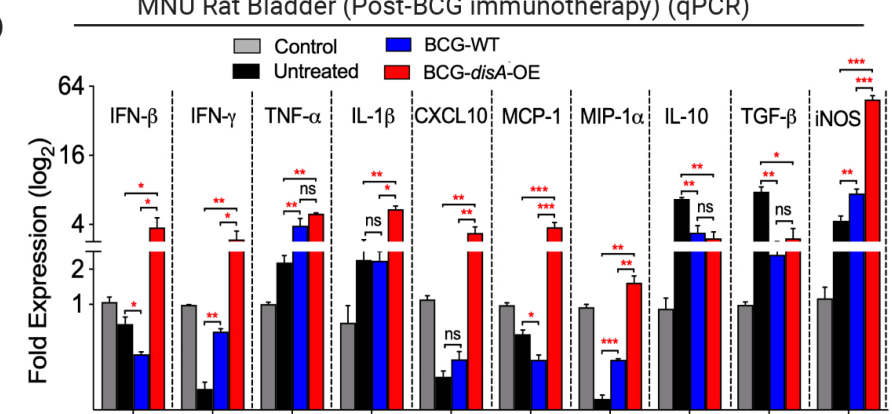

f

Ki67 Staining (MNU Rat Bladder)

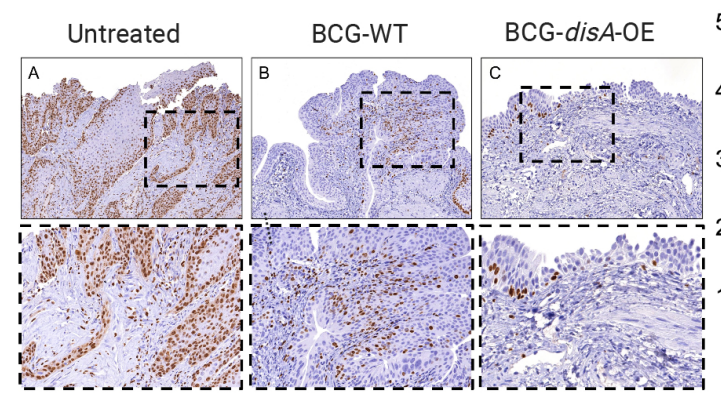

g CD68, CD86 and CD206 Staining (MNU Rat Bladder)

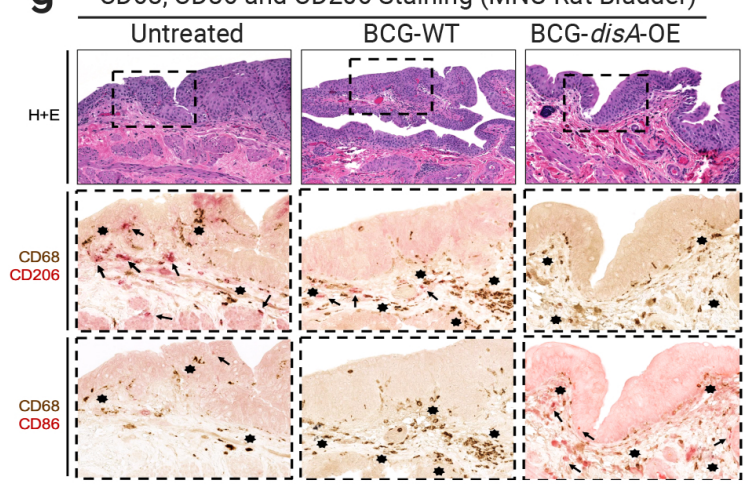

Ki67 IHC (MNU Rat Bladder)

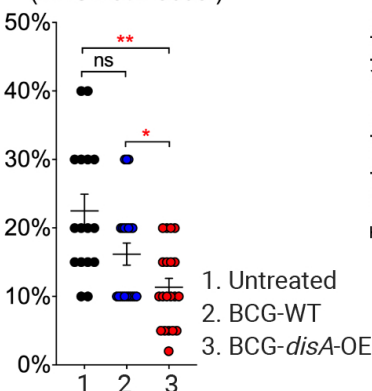

C MNU Rat Bladder (Post-BCG Immunotherapy)
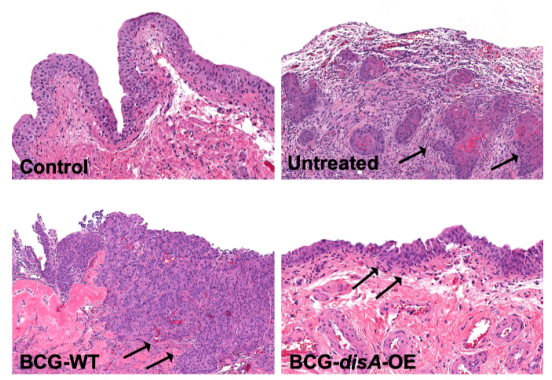

MNU Rat Bladder (Post-BCG Immunotherapy)

\begin{tabular}{|c|c|c|}
\hline Tumor Involvement Index & \multicolumn{2}{|c|}{ Cancer Staging } \\
\hline 1. Untreated & & $\square$ T2 \\
\hline 2. BCG-WT & p & $\square \mathrm{T} 1$ \\
\hline 3. BCG-disA-OE & c & a $\mathrm{Cls}$ \\
\hline$* * *$ & & $\square$ Normal \\
\hline
\end{tabular}
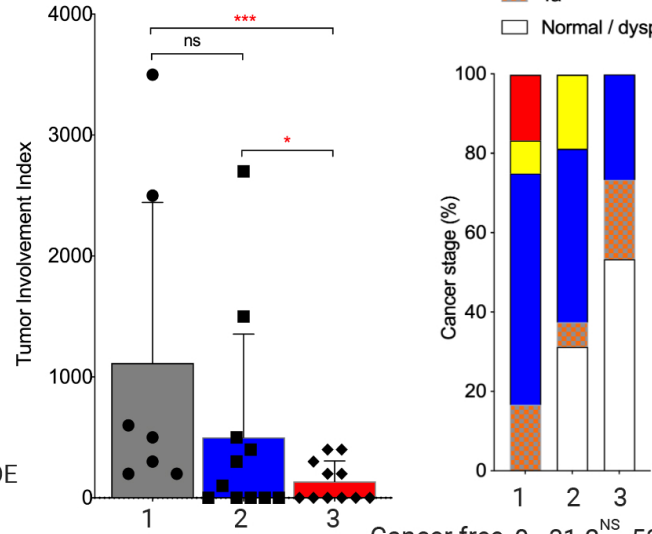

Cancer free $0 \quad 31.2^{\mathrm{NS}} 53.3^{\# \#}$

(\%)
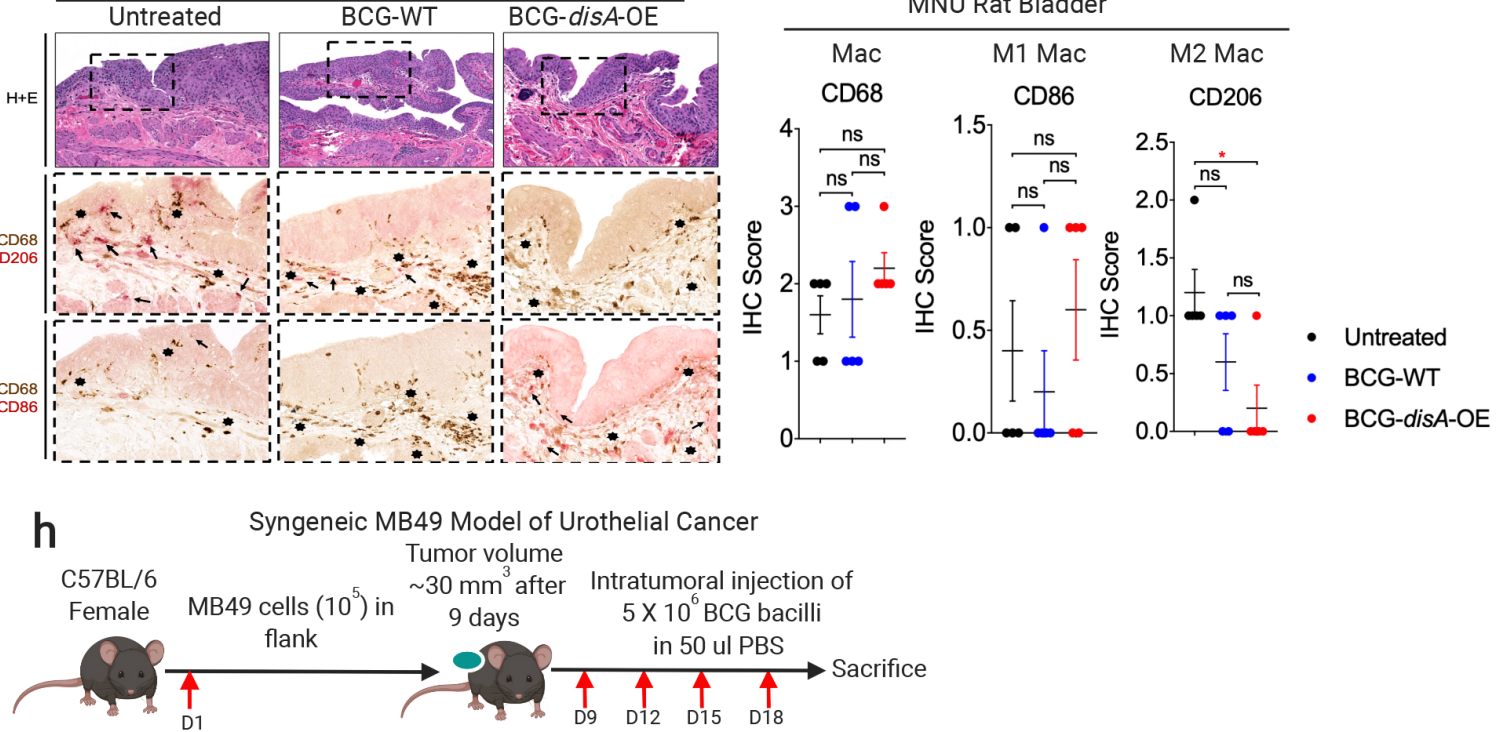

i Syngeneic MB49 Model of Urothelial Cancer

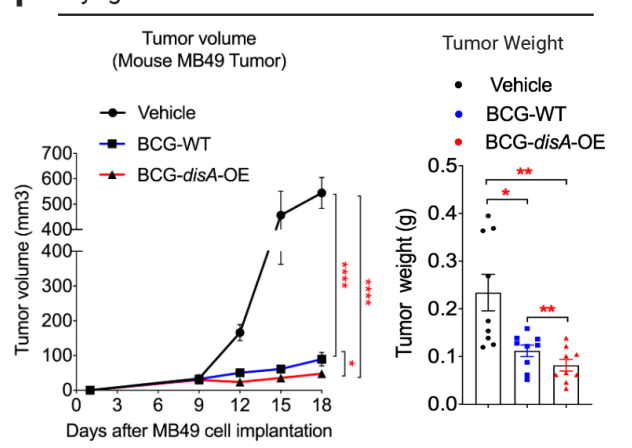

Syngeneic MB49 Model of Urothelial Cancer (Tumor)
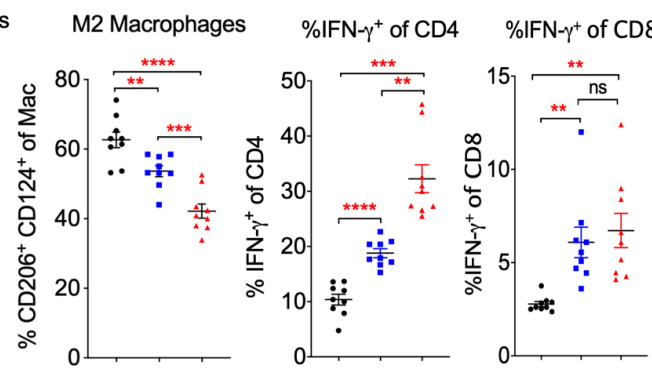
bioRxiv preprint doi: https://doi.org/10.1101/2020.04.25.061531; this version posted May 13, 2020. The copyright holder for this preprint (which was not certified by peer review) is the author/funder, who has granted bioRxiv a license to display the preprint in perpetuity. It is made Figure 1 available under aCC-BY 4.0 International license.

(Figure 1. continued) a. Schematic diagram of the MNU rat model of NMIBC. b. mRNA levels for proinflammatory cytokines (IFN- $\beta$, IFN- $\gamma$, TNF- $a$, and IL-1 $\beta$ ), regulatory chemokines (CXCL10, MCP-1, MIP-1a), immunosuppressive M2 macrophage cytokines (IL-10 and TGF- $\beta$ ) and M1 tumoricidal effectors (iNOS) in while bladders at necropsy (wk 23) measured by qRT-PCR relative to GAPDH ( 5 animals/group). c. Representative H \& E staining showing highest pathology grade for each group [control, untreated MNU bladder, BCG-WT (Past and Tice), and BCG-disA-OE (Past and Tice) -12-16 animals/group]. d. Tumor involvement values at necropsy (7-11 animals/group). e. Highest tumor stage at necropsy. f. Representative immunohistochemistry and bar graph of rat bladder tissue at necropsy stained for Ki67. g. Representative immunohistochemical co-staining and line graph for CD68 (brown), CD86 (M1 macrophages; red) and CD206 (M2 macrophages; red) in rat bladder tissue at necropsy; h. Schematic diagram of the MB49 syngeneic mouse model of urothelial cancer. i. MB49 tumor volume and tumor weight at the time of necropsy ( 9 animals/group). j. Tumor infiltrating M1 and M2 macrophages and CD4 and CD8 T cell lymphocyte types at necropsy in the MB49 model. ${ }^{\star} p<0.05,{ }^{\star \star} p<0.01,{ }^{\star \star \star} p<0.001,{ }^{\star \star \star \star} p<0.0001$ by 2-tailed Student's t-test. $\#$ \#p $<0.01$ by 1-way ANOVA. 
bioRxiv preprint doi: https://doi.org/10.1101/2020.04.25.061531; this version posted May 13, 2020. The copyright holder for this preprint (which was not certified by peer review) is the author/funder, who has granted bioRxiv a license to display the preprint in perpetuity. It is made Figure. 2 available under aCC-BY 4.0 International license.

a

BCG-disA-OE

(c-di-AMP overexpressing BCG)

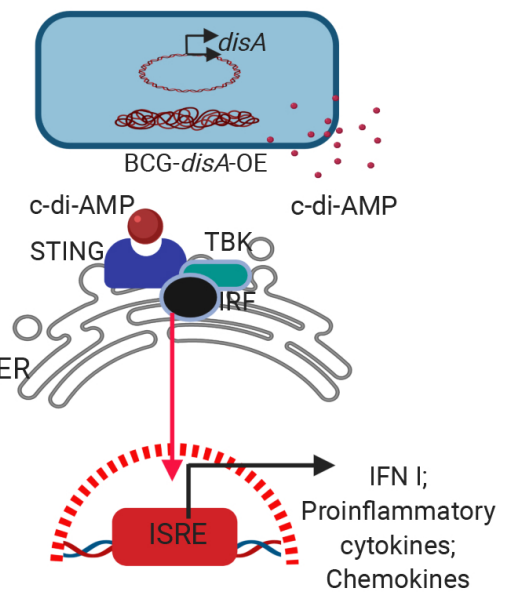

b

RAW-Lucia'T ISG

Reporter cells

$(1: 20 \mathrm{MOI})$

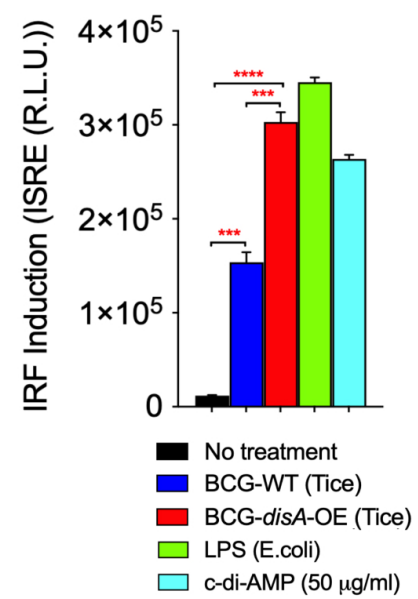

C IFN- $\gamma$ BMDM (M.O.I.: 1:20I)

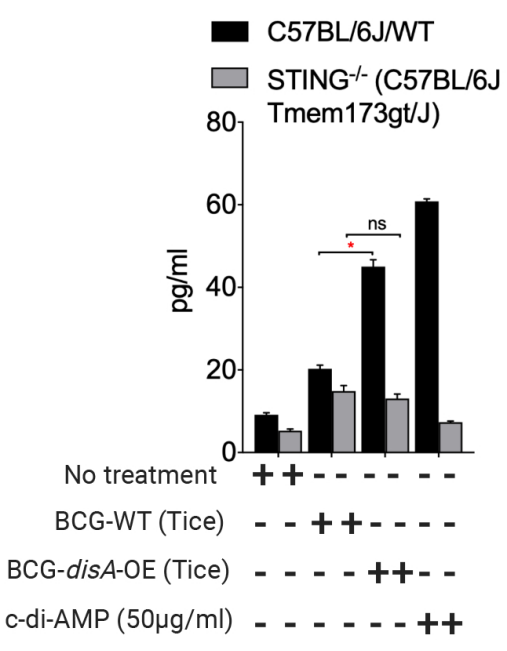

d M.O.I. $(1: 20)$
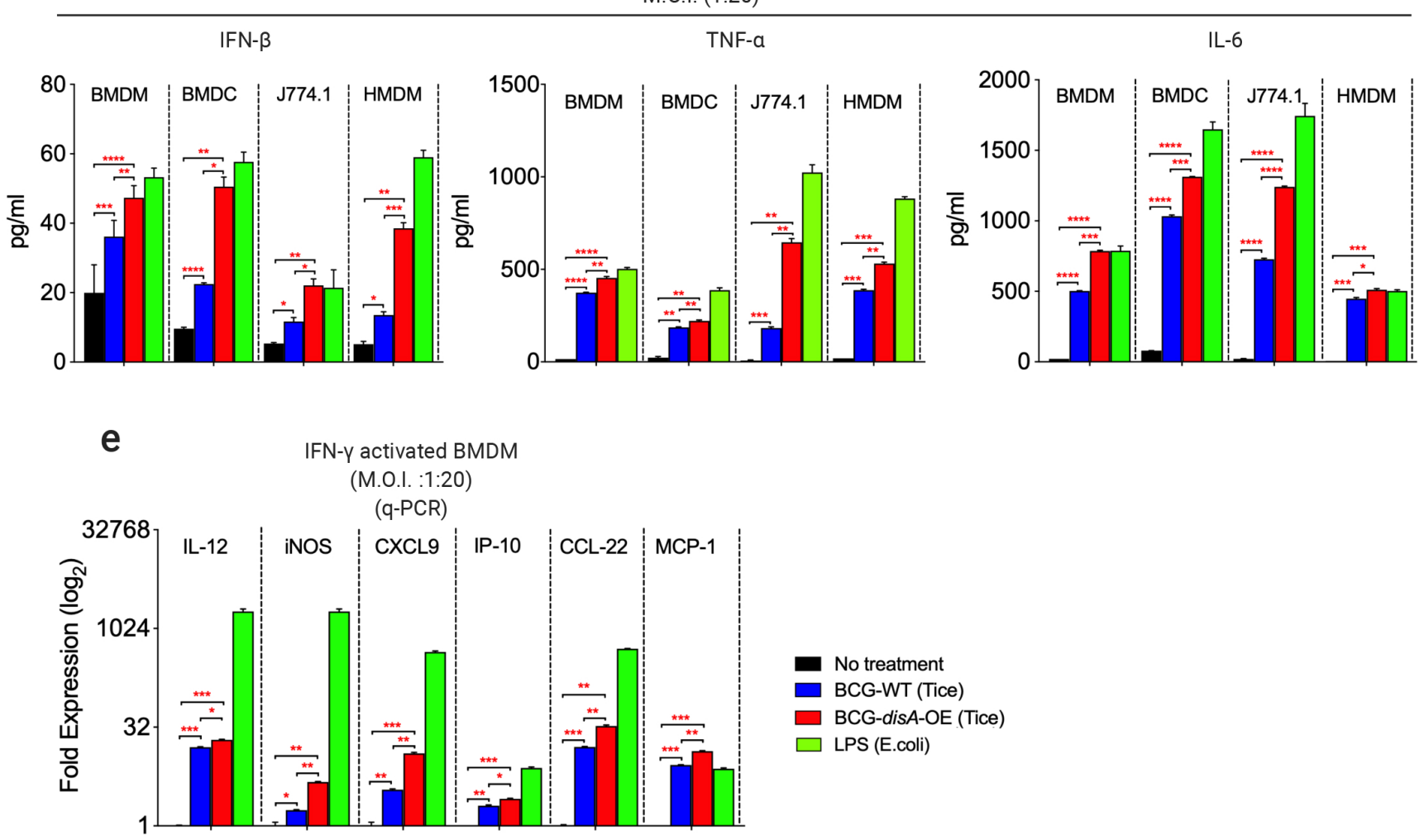

Figure 2. BCG- disA-OE elicits greater pro-inflammatory cytokine responses than BCG-WT in primary human and murine macrophages and dendritic cells in vitro. a. Schematic diagram. b. IRF3 induction measured in RAW-Lucia ISG reporter macrophages c. IFN- $\beta$ levels in murine BMDM from wild type and STING ${ }^{-/}$mice. d. IFN- $\beta$, TNF-a, and IL-6 levels from primary human and murine macrophages and dendritic cells and the J774.1 murine macrophage cell line. e. mRNA levels for Th1 cytokines and chemokines in murine BMDM relative to $\beta$-actin. Cytokine levels were measured by ELISA after $24 \mathrm{hr}$ exposures at a MOI of 20:1. Data are SEM $\left(n=3\right.$ replicates). ${ }^{*} p<0.05, * \star p<0.01, * \star * p$ $<0.001{ }^{\star \star \star \star \star} p<0.0001$ by 2-tailed Student's t-test. Data shown are for BCG-Tice; similar findings were observed for BCG-Pasteur as shown in Fig. S4. 
bioRxiv preprint doi: https://doi.org/10.1101/2020.04.25.061531; this version posted May 13, 2020. The copyright holder for this preprint (which was.not certified by peer review) is the author/funder, who has granted bioRxiv a license to display the preprint in perpetuity. It is made rigure.3 available under aCC-BY 4.0 International license.

a MOI (20:1)

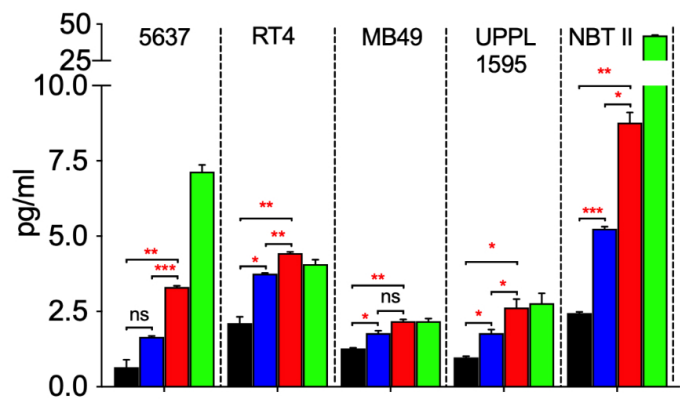

C

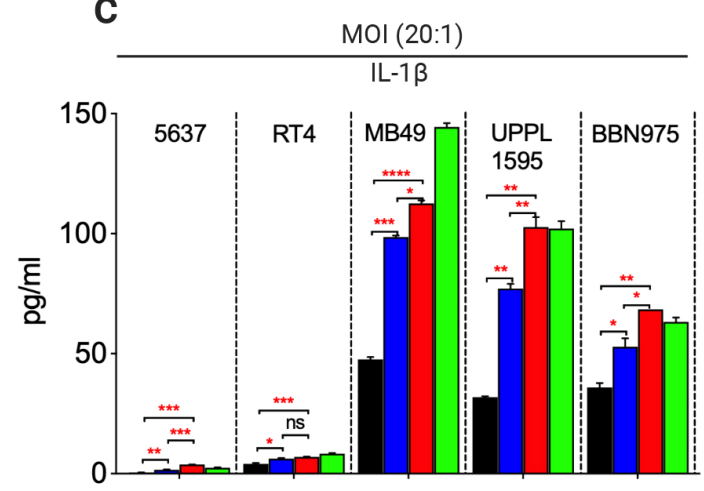

b
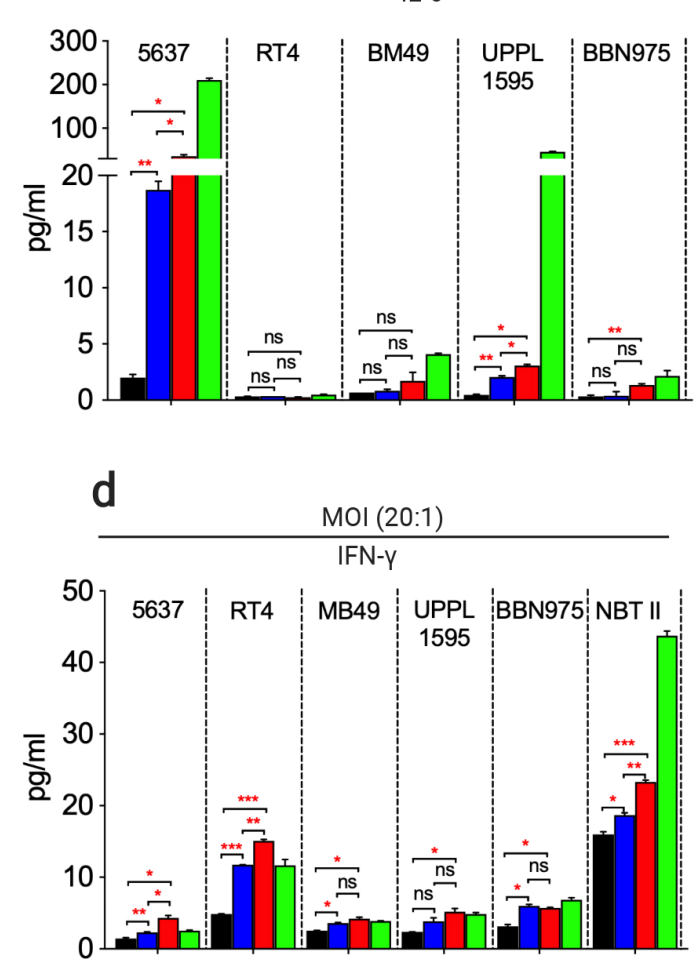

No treatment

- BCG-WT (Tice)

$\square$ BCG-disA-OE (Tice) $\square$ LPS (E.coli)

e

MOI (10:1)

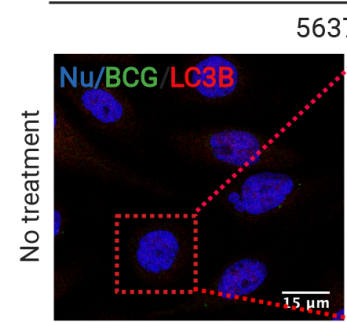

5637 Cells
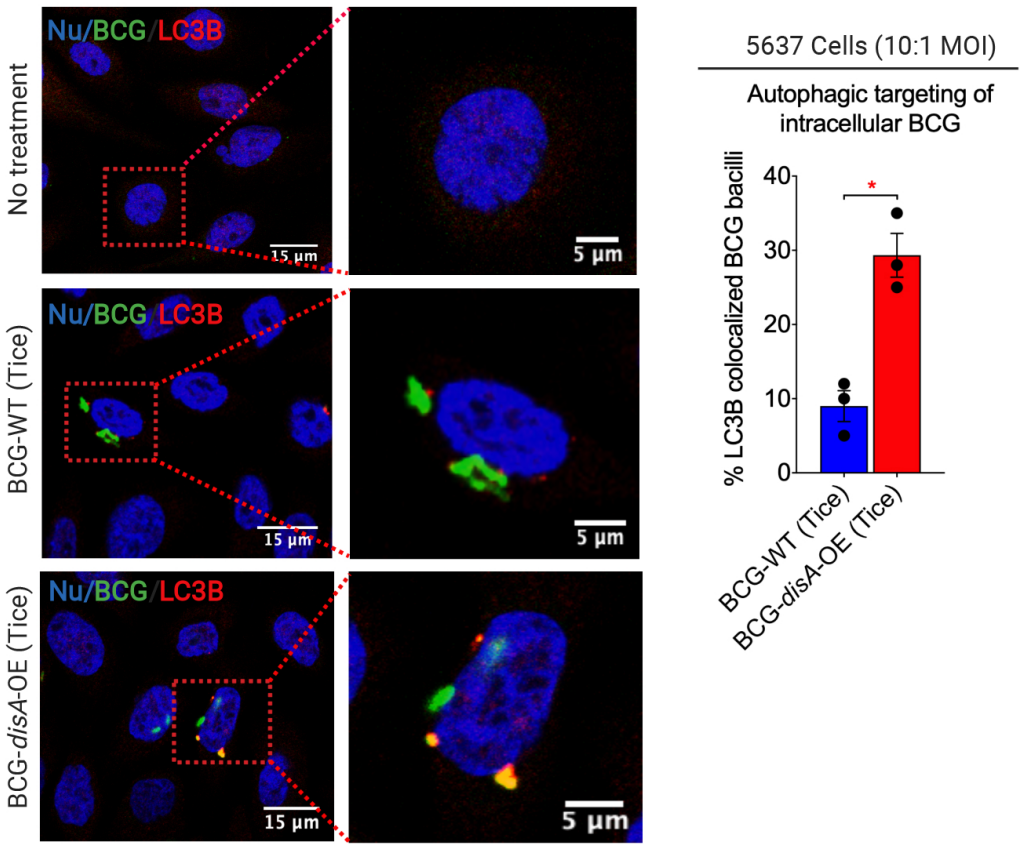

Figure 3. BCG- disA-OE elicits greater pro-inflammatory cytokine responses and autophagy than BCG-WT in human and murine urothelial carcinoma cells. a-d. TNF- $a, \mathrm{IL}-6, \mathrm{IL}-1 \beta$ and IFN- $\gamma$ levels in urothelial carcinoma cell lines: 5637 (human epithelial high grade carcinoma), RT4 (human transitional low grade carcinoam cells), NBT II (carcinogen-induced rat tumor), MB49, UPPL1595, and BBN975 (all carcinogen-induced mouse tumors). Cytokine levels were measured by ELISA after $24 \mathrm{hr}$ exposures at $\mathrm{MOI}$ of 20:1. Data are SEM ( $n=3$ replicates). e. Autophagy induction in the 5637 human urothelial carcinoma cells in representative confocal photomicrographs. Colocalization of FITC-labeled BCG strains (green), LC3B autophagic puncta (red) appears in yellow; nuclei are blue. Cells were fixed using $4 \%$ paraformaldehyde $3 \mathrm{~h}$ after infection (MOI 10:1), and images obtained with an LSM700 confocal microscope and Fiji software processing. ${ }^{*} p<0.05$, ${ }^{\star \star} p<0.01$, ${ }^{\star \star \star} p<0.001$, ${ }^{\star \star \star \star} p<0.0001$ by 2-tailed Student's t-test. Data shown are for BCG-Tice; similar findings were observed for BCG-Pasteur as shown in Fig. S7. 
bioRxiv preprint doi: https://doi.org/10.1101/2020.04.25.061531; this version posted May 13, 2020. The copyright holder for this preprint (which

Figlal\$ofcertified by peer review) is the author/funder, who has granted bioRxiv a license to display the preprint in perpetuity. It is made

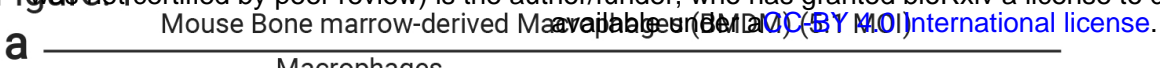
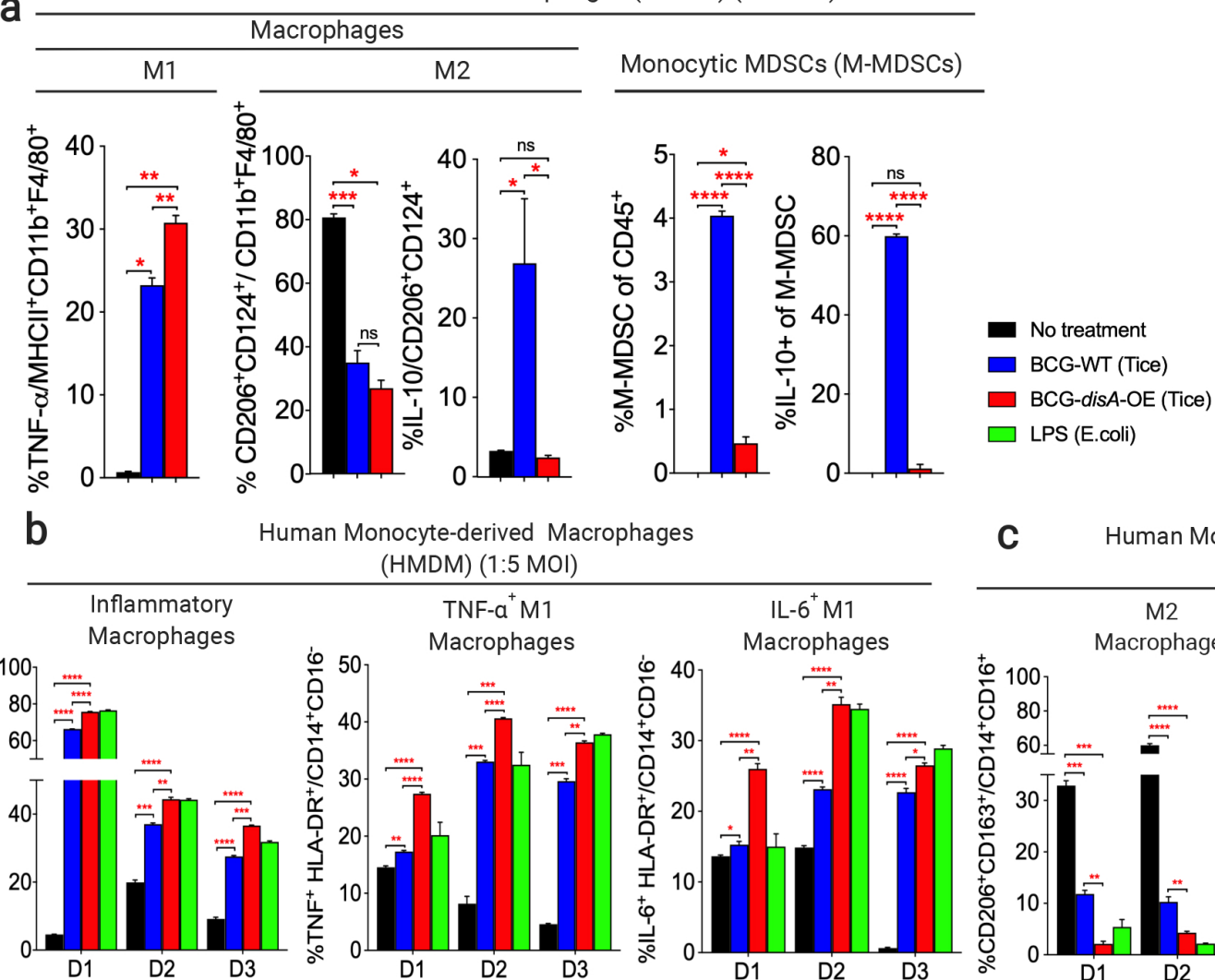

Human Monocyte-derived Macrophages (HMDM) (1:5 MOI)
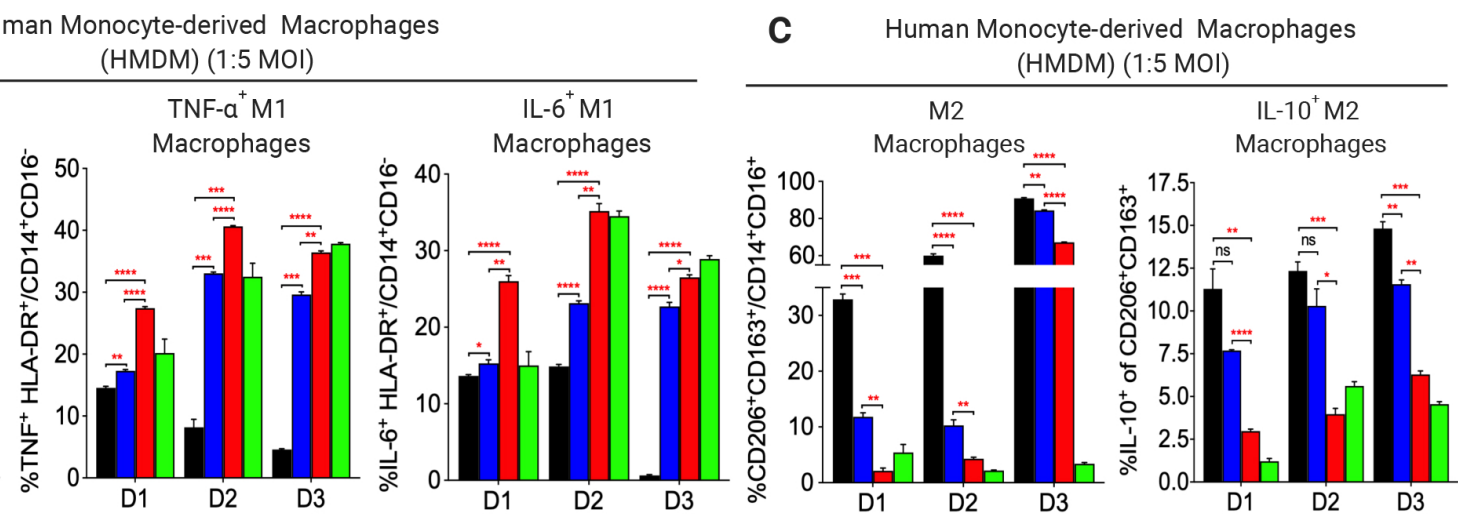

d

e

Phagocytic activity ( Human Monocyte)

IgG-FITC latex Bead Uptake
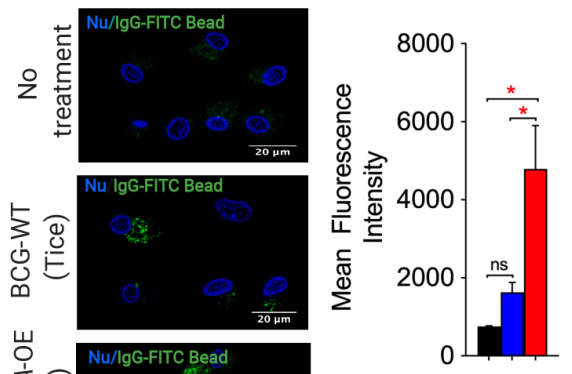

treatment

- BCG-WT (Tice)

BCG-disA-OE (Tice)
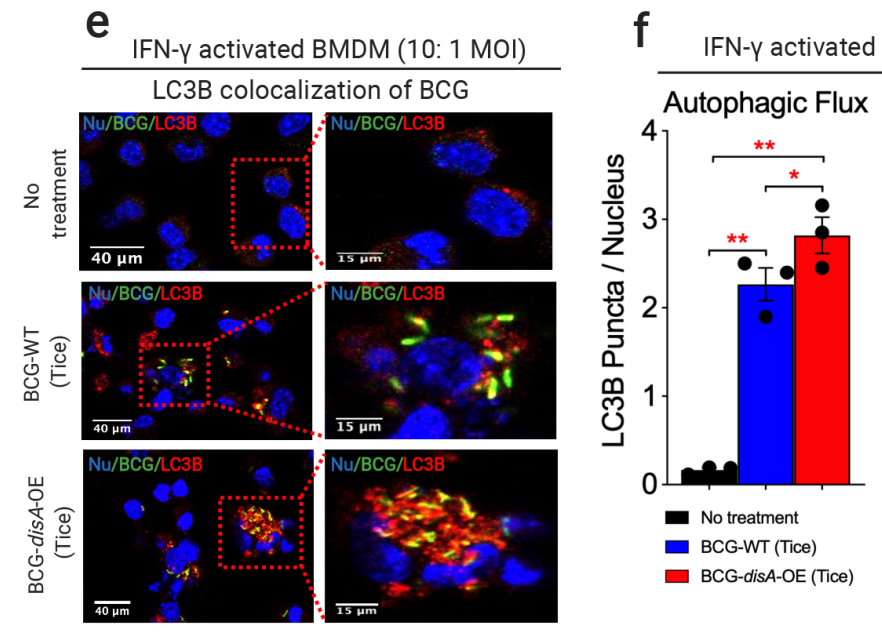

$\mathrm{h}$
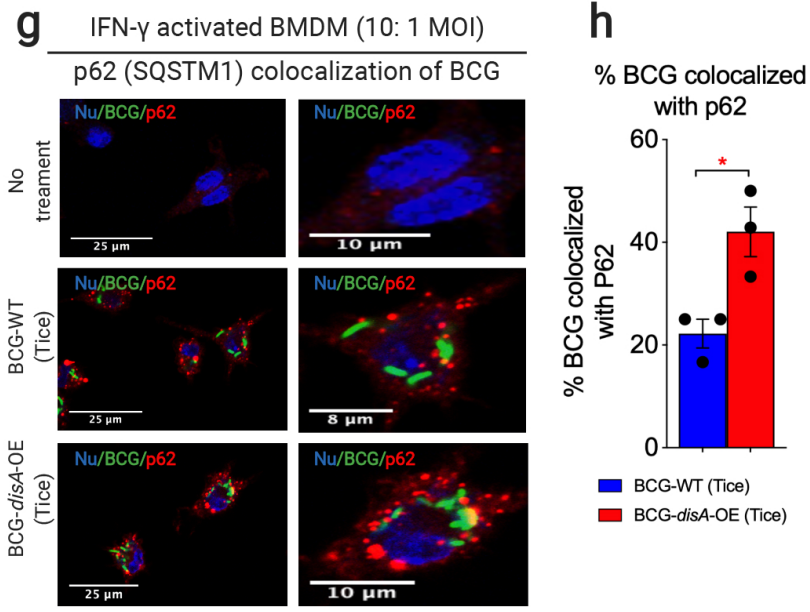

Figure 4. BCG- disA-OE elicits greater macrophage reprogramming, phagocytic activity, and autophagy than BCG-WT in human and murine macrophages. a. Percentage of M1- and M2-macrophages. b. Percetnages of inflammatory, TNF-a ${ }^{+} \mathrm{M} 1$, and IL-6+ M1 macrophages, and c. M2 and IL-10+ M2 macrophages arising (Continued). 
bioRxiv preprint doi: https://doi.org/10.1101/2020.04.25.061531; this version posted May 13, 2020. The copyright holder for this preprint (which

was not certified by peer review) is the author/funder, who has granted bioRxiv a license to display the preprint in perpetuity. It is made available under aCC-BY 4.0 International license.

\section{Figure.4 continued}

(Figure 4 continued) from primary human macrophages. Data were collected after $24 \mathrm{hr}$ exposures at MOI of 20:1 as determined by flow cytometry using gating schemes shown in Fig. S8-S13. Data are SEM ( $\mathrm{n}=3$ replicates). $d$. Phagocytic activity in human primary macrophages in representative confocal photomicrographs showing intracellular uptake of FITC-labeled IgG-opsonized latex beads (green) with nuclei stained blue. e. Autophagy induction and $\mathrm{f}$. quantification by BCG-LC3B colocalization in primary murine macrophages shown by representative confocal photomicrographs. Autophagy was measured by LC3B puncta or g. p62 colocalization with BCG appearing in yellow. FITC-labeled BCG strains are stained green, LC3B or p62 autophagic puncta (red), and nuclei blue. $h$. Quantification of BCG-p62 colocalization. Cells were fixed using 4\% paraformaldehyde $6 \mathrm{~h}$ after infection (MOI 10:1), and images obtained with an LSM700 confocal microscope and Fiji software processing. Quantification was by

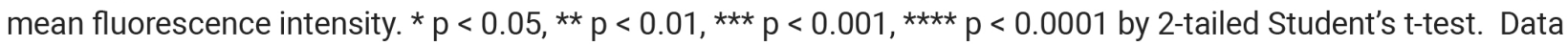
shown are for BCG-Tice; similar findings were observed for BCG-Pasteur as shown in Fig. S9 and S11. 
bioRxiv preprint doi: https://doi.org/10.1101/2020.04.25.061531; this version posted May 13, 2020. The copyright holder for this preprint (which Fig. 5 was not certified by peer review) is the author/funder, who has granted bioRxiv a license to display the preprint in perpetuity. It is made

a

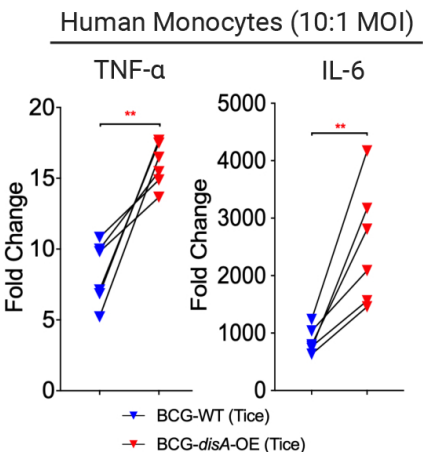

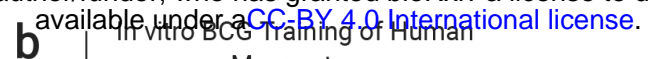

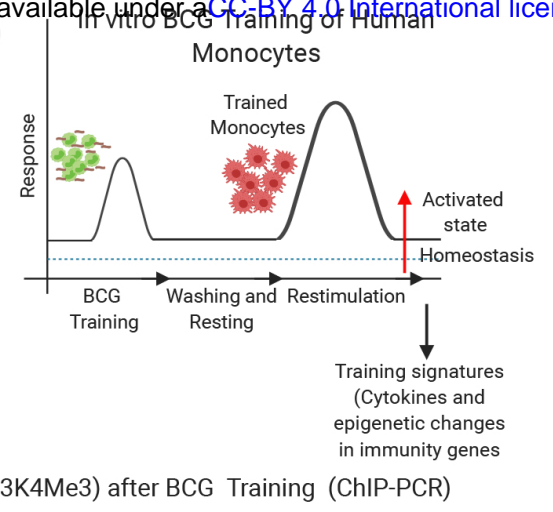

C

Chromatin Activation Mark (H3K4Me3) after BCG Training (ChIP-PCR)

Human Monocytes (Healthy Donors) (In vitro BCG Training)
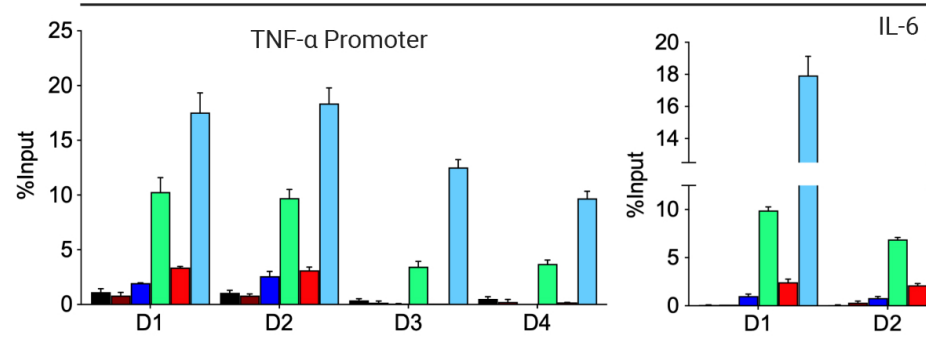

L-6 Promoter

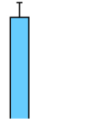

d Chromatin Repression Mark (H3K9Me3) post BCG Training (ChIP-PCR) Human Monocytes (Healthy Donors)
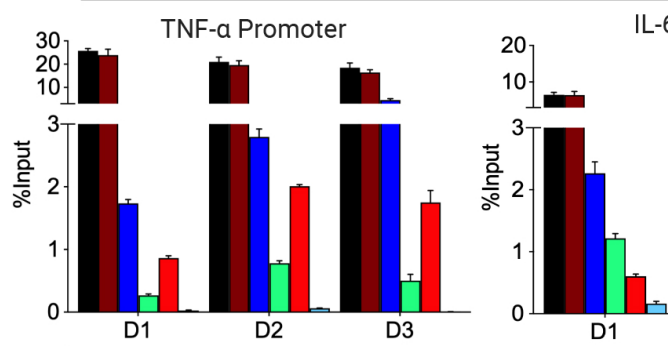

IL-6 Promoter
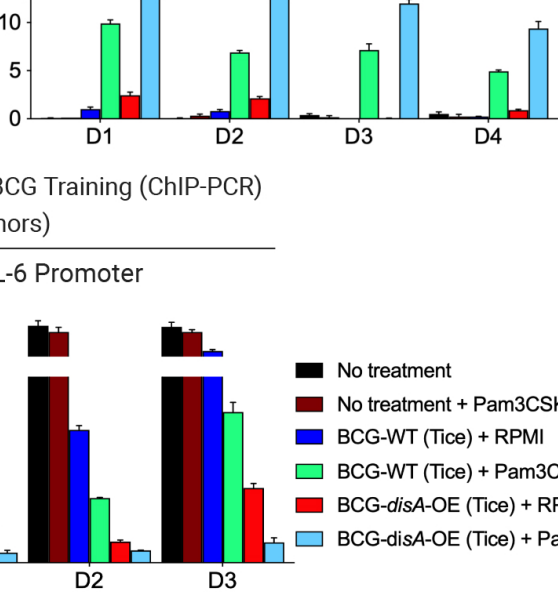

e

TNF-a Human Monocytes after BCG Training and Stimulation

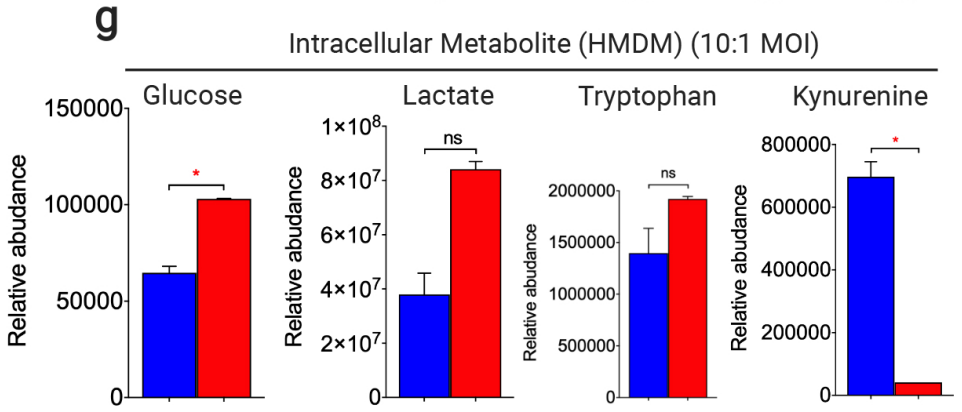

h Intracellular Metabolite (BMDM) (10:1 MOI)
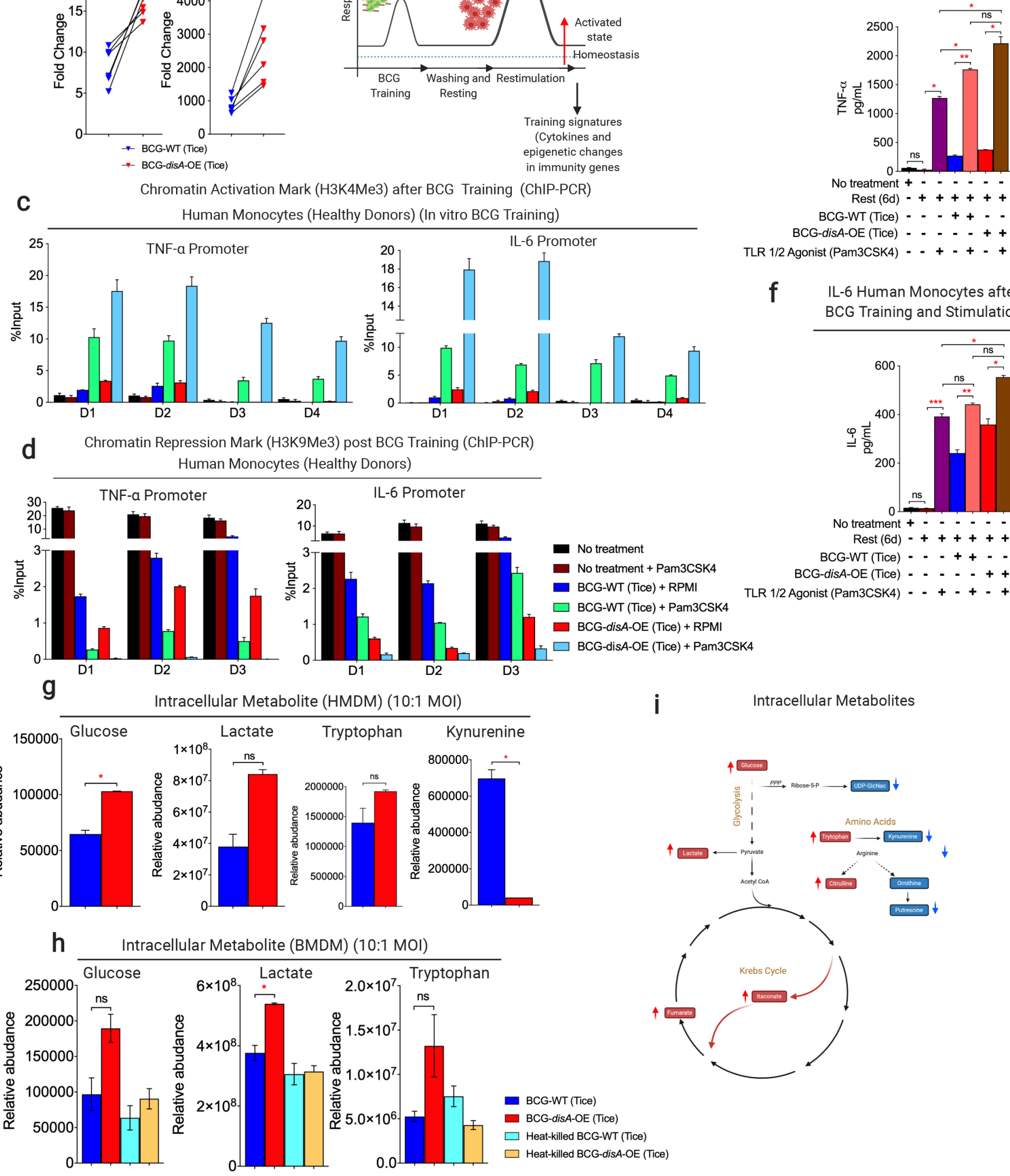

IL-6 Human Monocytes after BCG Training and Stimulation

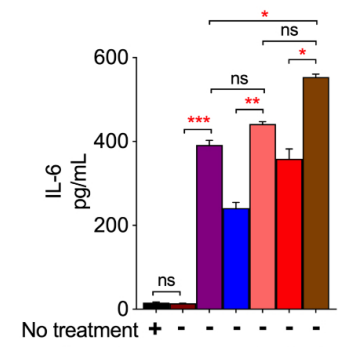

Rest $(6 \mathrm{~d})-++++++$

BCG-WT (Tice) - - - + + - -

BCG-disA-OE (Tice) - - - - - + +

TLR 1/2 Agonist (Pam3CSK4) - - + - + ++

BCG-WT (Tice) + Pam3CSK

- BCG-disA-OE (Tice) + RPMI

BCG-disA-OE (Tice) + Pam3CSK4

Intracellular Metabolites

Figure 5. Compared with BCG-WT, BCG- disA-OE is a more potent inducer of epigenetic and metabolomic changes characteristic of trained immunity in primary human monocytes. a. mRNA levels of TNF- $a$ and IL- 6 in primary human monocytes ( 6 healthy donors) relative to RNU6A after $24 \mathrm{hr}$ exposures at a $\mathrm{MOI}$ of 10:1. b. Schematic diagram of ex vivo monocyte training. c. Relative levels of the H3K4me3 chromatin activation mark or (d) the H3K9me3 chromatin repression mark retrieved from the TNF- $a$ and IL-6 promoter regions of primary human monocytes from 4 healthy donors (D1-D4) determined by ChIP-PCR assay on day 7 . e-f. Secreted cytokines (TNF- $a$ and IL-6) following BCG training and re-stimulation. Monocytes were initially challenged on day 0 with a $24 \mathrm{hr}$ exposure to the BCG strains at a MOI of 10:1 followed by washing. After five days of rest they were treated for $24 \mathrm{~h}$ with either a sham second stimulus (RPMI) or the TLR1/2 agonist Pam3CSK4. g-i. Metabolite levels determined by LCMS in human or murine MDM determined $24 \mathrm{hr}$ after exposure to BCG strains or heat-killed controls. Cartoon diagram (i) showing key metabolites upregulated (red arrow upward) in BCG-disA-OE infected macrophages relative to BCG-WT infected macrophages. $\quad * * p<0.01$ by 2-tailed Student's t-test. 
bioRxiv preprint doi: https://doi.org/10.1101/2020.04.25.061531; this version posted May 13, 2020. The copyright holder for this preprint (which Figul ne.6 certified by peer review) is the author/funder, who has granted bioRxiv a license to display the preprint in perpetuity. It is made

\section{a} available under aCC-BY 4.0 International license.

b
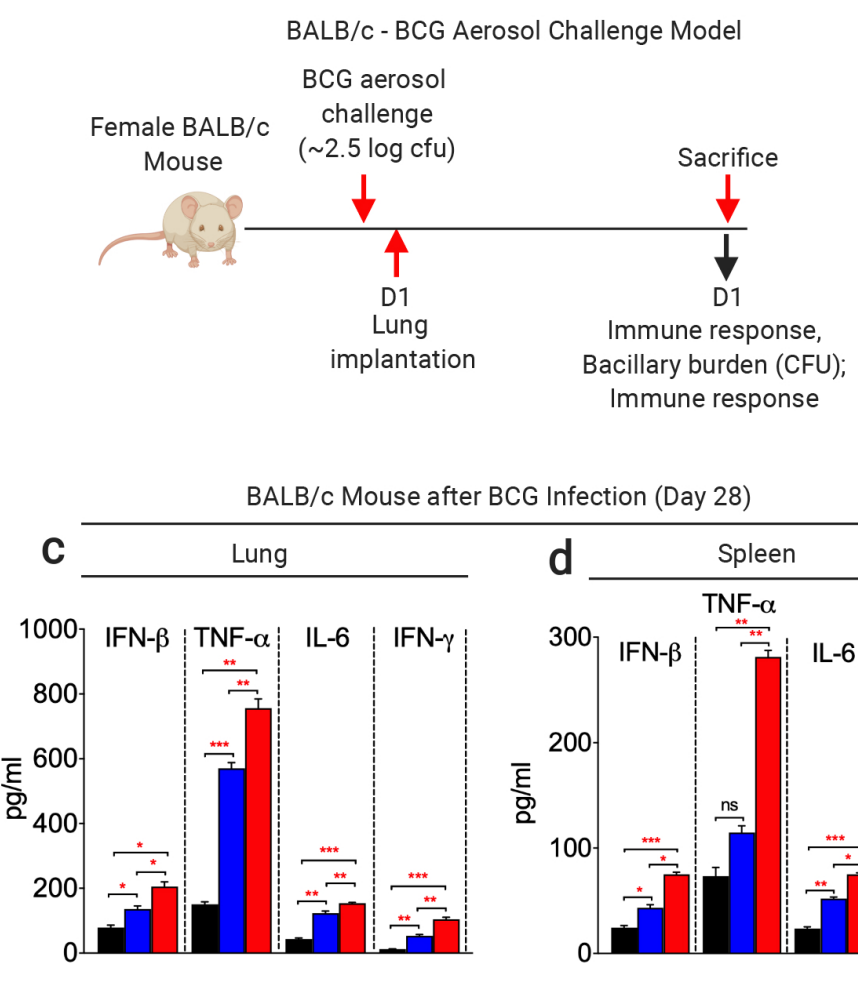

e

SCID - BCG Aerosol Challenge Model

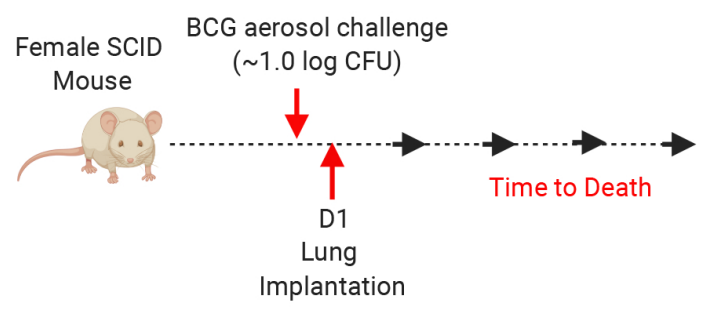

\section{Spleen}

f
Bacillary burden - BALB/C Mouse after BCG Infection (Attenuation of BCG-disA-OE in vivo)

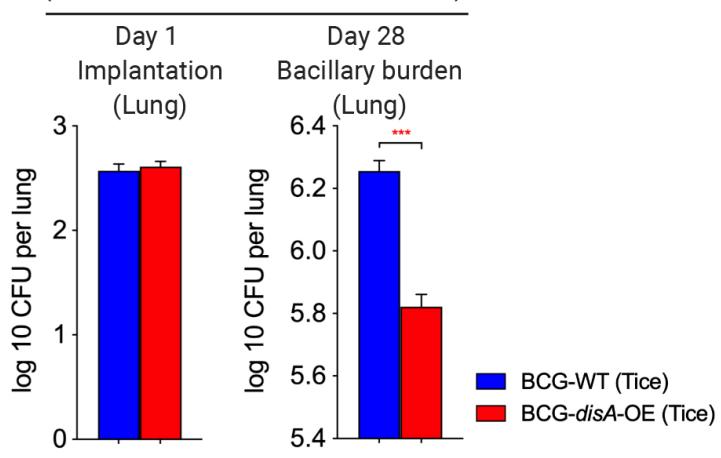

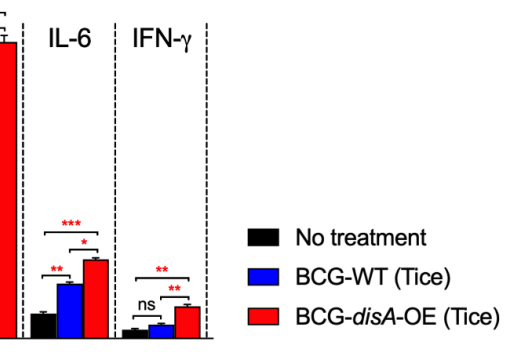

Time to Death (SCID Mice after BCG Infection)

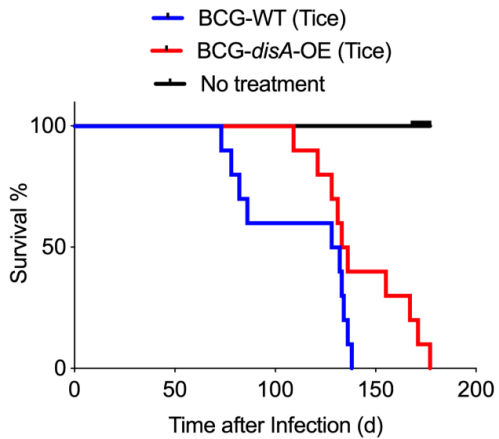

Bacillary burden - SCID Mouse after BCG Infection

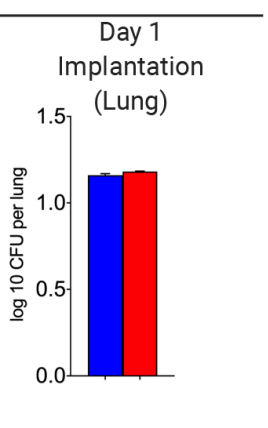

Figure 6. BCG- disA-OE is less pathogenic than BCG-WT in two mouse models and elicits a greater proinflammatory cytokine levels in vivo. a. Schematic diagram of the immunocompetent BALB/c mouse challenge model. b. Lung colony forming unit (CFU) counts at day 1 and day 28 ( 5 animals/group). Data are S.E.M. c. Levels of the pro-inflammatory cytokines IFN- $\beta$, TNF-a, IL-6, and IFN- $\gamma$ in mouse lungs and, $\mathrm{d}$. spleens determined by ELISA at day 28 (4 animals/group). Data are S.E.M. e. Schematic diagram of the immunocompromised SCID mouse challenge model. f. Percent survival of SCID mice following low dose challenge 10 animals/group). The day 1 lung CFU counts are shown at right. ${ }^{\star} p<0.05,{ }^{\star \star} p<0.01,{ }^{\star \star \star} p<0.001,{ }^{\star \star \star \star} p<0.0001$ by 2 -tailed Student's t-test. 